Kobayasi, K., Kobayashi, Y. and Oharu, S.

Osaka J. Math.

21 (1984), 281-310

\title{
NOLINEAR EVOLUTION OPERATORS IN BANACH SPACES
}

\author{
Kazuo KOBAYASI, Yoshikazu KOBAYASHI and ShinNosuke OHARU
}

(Received February 21, 1983)

\section{Introduction}

In this paper we discuss nonlinear evolution operators associated with the system of nonlinear evolution equations in a real Banach space $X$

$$
u^{\prime}(t) \in A_{t} u(t), \quad s<t<T,
$$

with initial conditions

$$
u(s)=x, \quad x \in \overline{D\left(A_{s}\right)} .
$$

In the above system, $0 \leqq s<T ; u(\cdot)$ stands for an $X$-valued unknown function on the interval $[s, T] ; u^{\prime}(\cdot)$ represents a derivative (perhaps in a generalized sense) of $u$; and $\left\{A_{t}: t \in[0, T]\right\}$ is a given family of nonlinear, possibly multivalued operators in $X$.

Suppose for the moment that there is a family $\left\{C_{s}: s \in[0, T]\right\}$ of subsets of $X$, and that for every $s \in[0, T)$ and every $x \in C_{s}$ the initial-value problem (hereafter called IVP) for $(D E)_{s}$ with $(I C)_{s}$ has a unique exact solution $u(\bullet ; s, x)$ on $[s, T]$ such that $u(t ; s, x) \in C_{t}$ for $s \leqq t \leqq T$. Then one can define operators $U(t, s)(0 \leqq s \leqq t \leqq T)$ in $X$ by

$$
U(t, s) x=u(t ; s, x) \quad \text { for } \quad x \in C_{s} \quad \text { and } \quad t \in[s, T] .
$$

The operator $U(t, s)$ maps $C_{s}$ into $C_{t}$ and has the following properties:

$$
U(s, s)=I / C_{s} \text { and } U(t, s) U(s, r)=U(t, r) \text { on } C_{r}
$$

for $0 \leqq r \leqq s \leqq t \leqq T$, where $I / C_{s}$ denotes the identity operator on $C_{s}$; and

(E2) for $s \in[0, T)$ and $x \in C_{s}, U(\cdot, s) x$ is strongly continuous on $[s, T]$.

In the case where $C_{s}$ is independent of $s$ and $C_{s} \equiv C$, a stronger continuity of $U(t, s)$ with respect to the parameters $s$ and $t$ may be obtained, namely:

$(\mathbf{E} 2)^{\prime}$ for $x \in C, U(t, s) x$ is strongly continuous over the triangle $0 \leqq s \leqq$ $t \leqq T$ with respect to $(s, t)$. 
Moreover, if the solution $U(\bullet ; s, x)$ depends continuously upon initial data $x \in C_{s}$ one will have that each $U(t, s)$ is continuous on $C_{s}$.

In this paper a family $\mathcal{U}$ of continuous operators $U(t, s)$ having properties (E1) and (E2) is called an evolution operator constrained in $\left\{C_{s}\right\}$. If in particular $C_{s}$ is constant and $C_{s} \equiv C$, the family $\mathcal{U}$ of continuous operators with properties (E1) and (E2)' is called an evolution operator on $C$. In what follows, we are mainly concerned with evolution equations in nonreflexive Banach spaces; and in this case, it should be noted that a nonlinear equation $(D E)_{s}$ does not necessarily have exact solutions even if the $t$-dependence of $A_{t}$ is smooth and initial-data are nice. However, if $\mathcal{U}$ arises in such a way that $U(t, s) x$ gives a solution (in a generalized sense) to the IVP for $(D E)_{s}$ and $\mathcal{U}$ has properties (E1) and (E2), such a family $\mathcal{U}$ is called an evolution operator associated with the system $\left\{(D E)_{s}\right\}$.

The present paper is devoted to the studies of two problems below. The first aim of this paper is to introduce two notions of generalized solutions to $(D E)_{s}$ such that the associated solution operators form an evolution operator as mentioned above. Firstly we consider the case in which approximate differential or difference equations for $(D E)_{s}$ can be derived and there exist limits of solutions of those approximate equations. In this case it is relevant to regard the limits as solutions in a generalized sense and we treat generalized solutions of this kind (called herein weak solutions to $(D E)_{s}$ ).

On the other hand, $\mathrm{Ph}$. Bénilan introduced in [1] a notion of integral solution which is given in terms of specific integral inequalities. His notion plays an important role both in giving the framework of the theory of nonlinear contraction semigroups and in establishing the uniqueness theorem of such nonlinear semigroups generated by dissipative operators. In view of this, we employ a certain type of $t$-dependence of the operator $A_{t}$ in order to extend the notion of Benilan's integral solution to the time-dependent case under consideration, and then show that given an initial-value $x \in D\left(A_{s}\right)$, the associated weak solution is uniquely determined in the class of such extended integral solutions.

The next problem is to discuss the generation of an evolution operator that provides generalized solutions of the IVP. In 1967 Kato gave in [10] a general theory of nonlinear evolution operators in Banach spaces with uniformly convex duals and, in 1972, Crandall and Pazy [4] established a generation theory in general Banach spaces. Since that time many works concerning this problem have been published. See Crandall and Evans [5], Evans [8], Kobayashi [14], Pavel [25], and Kobayasi and Oharu [15]. Our second purpose of this paper is to discuss the construction of evolution operators which furnish the integral solutions of $(D E)_{s}$ mentioned as in the preceding paragraph and extend the results quoted above. 
0. Outline. Our argument contains three features. Firstly, we think of a proper lower semicontinuous functional $\mathfrak{p}: X \rightarrow[0,+\infty]$ to specify the $t$-dependence of $A_{t}$, the boundedness of solutions, etc.. Hence the $t$-dependence in our argument depends upon the choice of a functional $\mathfrak{p}$. In Section 1 we shall introduce a class of operators $A_{t}$ by means of a functional $\mathfrak{p}$ as above and discuss the $t$-dependence of $A_{t}$. In Section 2 we shall introduce a notion of integral solution for the time-dependent case in terms of the functional $\mathfrak{p}$ and establish a uniqueness theorem of Benilan's type. Some of interesting examples of the functionals as mentioned above are found in [9], [15], [22] and [23].

Secondly, we discuss the construction of evolution operators through the following type of discrete approximation of $(D E)_{s}$ : Let $s \in[0, T), x \in X$ the initial-value given in $(I C)_{s}$, and suppose that there is a system $\left(\left\{\Delta_{n}\right\},\left\{\left(u_{k}^{n}, v_{k}^{n}\right)\right\}\right.$, $\left.\left\{f_{k}^{n}\right\}\right)$ of sequences with the following properties:

(i) $\left\{\Delta_{n}\right\}$ is a sequence of partitions of $[s, T]$ of the form

$$
\Delta_{n}=\left\{s=t_{0}^{n}<t_{1}^{n}<\cdots<t_{N_{n^{+1}}}^{n}=T\right\}(n \geqq 1) \quad \text { and } \quad \lim _{n \rightarrow \infty}\left|\Delta_{n}\right|=0 .
$$

ii) $\left\{u_{k}^{n}\right\},\left\{v_{k}^{n}\right\}$ and $\left\{f_{k}^{n}\right\}$ are sequences in $X$ and satisfy the difference equation

$$
\begin{array}{ll}
((D E))_{n} \quad\left(h_{k}^{n}\right)^{-1}\left[u_{k}^{n}-u_{k-1}^{n}\right]=v_{k}^{n}+f_{k}^{n}, \quad\left(u_{k}^{n}, v_{k}^{n}\right) \in A_{t_{k}^{n}} \\
& h_{k}^{n}=t_{k}^{n}-t_{k}^{n-1}, \quad 1 \leqq k \leqq N_{n}
\end{array}
$$

for $n \geqq 1$ as well as the following condition

$$
\lim _{n \rightarrow \infty} u_{0}^{n}=x \quad \text { and } \quad \lim _{n \rightarrow \infty} \sum_{k=1}^{N_{n}} h_{k}^{n}\left\|f_{k}^{n}\right\|=0 .
$$

The system of difference equations $\left\{((D E))_{n}: n \geqq 1\right\}$ together with condition $(C)$ is regarded as an approximate discrete scheme for $(D E)_{s}$ with $(I C)_{s}$; and condition $(C)$ is understood to mean that the scheme is consistent with IVP for $(D E)$ with $(I C)$. In what follows, we denote such scheme by $((D S))_{s}$ and we say that $((D S))_{s}$ is a consistent discrete scheme for $(D E)_{s}$. Now defining step functions $u_{n}(t)=u_{n}(t ; s, x)$ by $u_{n}(s)=u_{0}^{n}$ and $u_{n}(t)=u_{k}^{n}$ for $t \in\left(t_{k-1}^{n}, t_{k}^{n}\right]$, the evolution operator $\mathcal{U}$ is obtained as $U(t, s) x=\lim u_{n}(t ; s, x)$. In Section 3 we shall employ a modified version of estimation due to Kobayashi [13] in order to obtain the convergence of $u_{n}$.

Thirdly, we shall construct evolution operators $\bigcup$ such that $U(t, s)$ is locally Lipschitz continuous on its domain. Employing a proper, lower semicontinuous functional $\mathfrak{p}$ on $X$, we consider the class of initial-data $x$ with $\mathfrak{p}(x)<\infty$ and discuss in Section 4 the construction of the evolution operators on each of the closed sets $X_{\alpha}=\{x: \mathfrak{p}(x) \leqq \alpha\}(\alpha>0)$; hence the evolution operator $\mathcal{V}$ is constructed on the effective domain $X_{0}=D(\mathfrak{p})$ of $\mathfrak{p}$ in such a way that each 
$U(t, s)$ satisfies

$$
\|U(t, s) x-U(t, s) y\| \leqq \exp \left[\omega_{a}(t-s)\right]\|x-y\|
$$

for $x, y \in X_{\alpha} \cap \overline{D\left(A_{s}\right)}, t \in[s, T]$, and some constant $\omega_{\alpha}$ depending upon $\alpha>0$.

Assumption of such a functional $\mathfrak{p}$ does not impose restrictions to the generation theory, but rather makes it possible to treat the evolution governed by more general operators than dissipative (or accretive) operators.

In fact, if we want to get a generation theorem (especially time-independent case) without such functionals, it is acquired by putting $\mathfrak{p}(x) \equiv 0$ on $X$ in our argument. The generation theorems established by Crandall and Pazy [4], Evans [8] and Pavel [25] are obtained by taking appropriate functionals $\mathfrak{p}$. For the time-independent case there are the recent works of Pierre [26, 27] in our direction; and our results extend his generation theorems to the timedependent case. Utility of making use of such functionals $\mathfrak{p}$ will also be illustrated in various applications of our results to concrete partial differential equations, which we shall treat in [15] and in the forthcoming papers [9] and [23].

The relations of our results to the other works quoted above will be discussed in Remarks contained in each section. There are two types of general hypotheses for the family of operators $\left\{A_{t}\right\}$ and both types of assumptions together extend most of the conditions treated in the works cited above. Here we treat the generation problem for evolution operators under the first type of hypotheses for $\left\{A_{t}\right\}$. The second type of assumption will be discussed in the forthcoming paper of the authors [18].

\section{Basic hypotheses}

In this section we make basic hypotheses on the family of operators $\left\{A_{t}\right.$ : $t \in[0, T]\}$ for which equations $(D E)_{s}$ are formulated and then discuss the $t$ dependence of $A_{t}$.

Let $X$ be a real Banach space with norm $\|\cdot\|$. By an operator $A$ in $X$ we mean a (possibly multi-valued) operator with domain $D(A)$ and range $R(A)$ in $X ; D(A)$ is the set $\{x \in X: A x \neq \emptyset\}$ and $R(A)$ is the union $\cup\{A x: x \in D(A)\}$. In this paper operators in $X$ are identified with their graphs in $X \times X$; hence we sometimes write $(x, y) \in A$ when $y \in A x$. For $x \in D(A)$ we write $\|\mid A x\| \|$ for the value inf $\{\|y\|: y \in A x\}$. Following Takahashi [30], we define an extended real-valued functional $|A \cdot|: X \rightarrow[0, \infty]$ by

$$
|A x|=\inf \left\{\sup _{n}\left\|\left|A x_{n}\right|\right\|: x_{n} \in D(A) \text { and } x_{n} \rightarrow x\right\} \quad \text { for } \quad x \in \overline{D(A)}
$$

and $|A x|=+\infty$ for $x \notin \overline{D(A)}$. We permit ourselves the common abbreviation, an l.s.c. functional on $X$, in referring to a lower semicontinuous functional on $X$. 
The functional $|A \cdot|$ is 1.s.c. on $X$ and its effective domain $D_{a}(A)=$ $\{x \in X:|A x|<\infty\}$ satisfies $D(A) \subset D_{a}(A) \subset \overline{D(A)}$. The set $D_{a}(A)$ is closely related to the notion of generalized domain introduced by Crandall [7]. We shall use the following fact: Given an $x \in D_{a}(A)$, there exists a sequence $\left\{x_{l}\right\}$ in $D(A)$ such that $x_{l} \rightarrow x$ in $X$ and $\left\|\left|A x_{l}\right|\right\| \leqq|A x|+1 / l$ for $l \geqq 1$. The closure of the operator $A$ means the operator defined by the closure in $X \times X$ of the graph of $A$ and is denoted by $\bar{A}$. The restriction of $A$ to a set $C$ is denoted by $A / C$.

Firstly, in order to specify the class of nonlinear operators with which we are concerned in this paper, we employ two sorts of tangent functionals of the unit ball of $X, \tau_{+}$and $\tau_{-}$on $X \times X$ defined respectively by

$$
\begin{aligned}
& \tau_{+}(v, u)=\lim _{t \downarrow 0} \frac{1}{t}(\|u+t v\|-\|u\|) ; \quad \text { and } \\
& \tau_{-}(v, u)=\lim _{t \downarrow 0} \frac{1}{t}(\|u\|-\|u-t v\|) \quad \text { for } \quad u, v \in X .
\end{aligned}
$$

Notice that the values $\tau_{ \pm}(v, u)$ are sometimes denoted by $\tau_{ \pm}(u, v)$, respectively. Since the norm $\|\cdot\|$ is convex on $X$, both $\tau_{+}$and $\tau_{-}$are well-defined on $X \times X$. Basic properties of these functionals are mentioned in [8], [19] and [20]. In particular, we shall use the following facts in later arguments:

$\left(1^{0}\right) \quad \tau_{-}(v, u)=-\tau_{+}(-v, u)$ for $u, v \in X$.

$\left(2^{\circ}\right)$ The duality mapping $F$ of $X$ is the subdifferential of the convex functional $u \rightarrow \frac{1}{2}\|u\|^{2}$ on $X$ and we have

$$
\begin{aligned}
& \tau_{+}(v, u)\|u\|=\sup \{\langle v, f\rangle: f \in F(u)\}, \\
& \tau_{-}(v, u)\|u\|=\inf \{\langle v, f\rangle: f \in F(u)\} .
\end{aligned}
$$

where $\langle v, f\rangle$ denotes the value of $f \in X^{*}$ at $v$.

$\left(3^{0}\right)$ The functional $(u, v) \rightarrow \tau_{+}(v, u)$ is upper semicontinuous on all of $X \times X$ with respect to the strong topology.

We shall also use tangent functionals on $X^{* *} \times X^{* *}$; but we denote them by the same symbols $\tau_{+}$and $\tau_{-}$since $X \times X$ can be regarded as a closed subspace of $X^{* *} \times X^{* *}$.

Secondly we use an 1.s.c. functional (denoted herein $\mathfrak{p})$ to classify the class of initial-data for the system of evolution equations $\left\{(D E)_{s}\right\}$. Let $\mathfrak{p}: X \rightarrow$ $[0, \infty]$ be an 1.s.c. functional on $X$ such that the effective domain $D(\mathfrak{p})$ intersects $D\left(A_{t}\right)$ for $t \in[0, T]$. We define

$$
X_{\infty}=\{x \in X: \mathfrak{p}(x) \leqq \alpha\} \quad \text { and } X_{\alpha, s}=X_{\infty} \cap D\left(A_{s}\right)
$$

for $\alpha>0$ and $s \in[0, T]$, and set $X_{0}=\cup\left\{X_{a}: \alpha>0\right\}$. The set $X_{\infty}$ is closed in $X$ 
and monotone increasing as $\alpha \uparrow+\infty$; and we have $X_{0}=D(\mathfrak{p})$. We sometimes write $X_{\infty}$ for the whole space $X$ for convenience of notation. It turns out that we treat the evolution equations in the system $(X,\|\cdot\|, \mathfrak{p})$, where the norm is for the convergence of elements of $X$ and the functional specifies the boundedness of elements under consideration.

Thirdly, in order to specify the $t$-dependence of $A_{t}$, we introduce two classes of functions on $[0, T] \times[0, T]$. A nonnegative bounded function $\theta$ defined on all of $[0, T] \times[0, T]$ is said to belong to the class $\mathscr{Z}$ if it satisfies the following two conditions:

(i) $\theta$ is upper semicontinuous on all of $[0, T] \times[0, T]$; and

(ii) $\theta$ is symmetric with respect to $(s, t), \theta(s, s)=0$ for $s \in[0, T]$, and

$$
\lim _{\delta \downarrow 0} \sup \{\theta(t, s):|t-s| \leqq \delta\}=0 .
$$

Note that if $\theta_{1}, \theta_{2} \in \mathcal{L}$ then $\theta_{1}+\theta_{2} \in \mathscr{L}$. If $\theta$ is continuous on $[0, T] \times[0, T]$, then $\theta \in \mathscr{L}$ iff $\theta$ is symmetric and $\theta(s, s)=0$ for $s \in[0, T]$.

Given a $\theta \in \mathscr{L}$, we define $\rho:[0, T] \rightarrow[0, \rho(T)]$ by

$$
\rho(r)=\sup \{\theta(t, s): s, t \in[0, T] \quad \text { and } \quad|t-s| \leqq r\} \quad \text { for } \quad r \in[0, T] \text {. }
$$

The function $\rho$ is bounded, nonnegative and nondecreasing on $[0, T]$, and $\lim _{r \downarrow 0} \rho(r)=0$. Hence we may assume that $\rho$ is right-continuous on $[0, T)$. If $\theta$ is continuous on $[0, T] \times[0, T]$, then $\rho$ is upper semicontinuous on $[0, T]$ and right-continuous on $[0, T)$. Conversely, if $\rho:[0, T] \rightarrow R$ is any bounded, nonnegative, nondecreasing and right-continuous function with $\lim _{r \downarrow 0} \rho(r)=\rho(0)$ $=0$, then the function $\theta$ defined by $\theta(t, s)=\rho(|t-s|)$ for $(t, s) \in[0, T] \times[0, T]$ belong to the class $\mathcal{I}$.

A function $\theta$ defined on all of $[0, T] \times[0, T]$ is said to belong to the class $\mathcal{I}_{B V}$, if $\theta \in \mathscr{I}$ and the following additional condition holds:

(iii) there exists a constant $M>0$ such that

$$
\sum_{k=1}^{N} \theta\left(t_{k}, t_{k-1}\right) \leqq M
$$

for all partitions $\Delta=\left\{0=t_{0}<t_{1}<\cdots<t_{N}=T\right\}$ of $[0, T]$.

We now make basic hypotheses on the family of operators $\left\{A_{t}\right\}$ by using the above terminology. First the following condition is always assumed whenever a functional $\mathfrak{p}$ as mentioned above is considered:

(P) For every $\beta>0$ there is an $\alpha \geqq \beta$ such that $X_{\beta} \cap \overline{D\left(A_{s}\right)} \subset \bar{X}_{\alpha, s}$ for $s \in[0, T]$.

Practically, the functional $\mathfrak{p}$ represents various kinds of stability for the operators and is suitably chosen so that condition $(\boldsymbol{P})$ holds. See for instance [9], [15], [22] and [23]. Condition $(\boldsymbol{P})$ yields the relation 


$$
C_{s}=X_{0} \cap \overline{D\left(A_{s}\right)}=\bigcup_{\beta>0} \bar{X}_{\beta, s} \quad \text { for } \quad s \in[0, T] .
$$

Under condition $(\boldsymbol{P})$ we assume throughout this paper that the domain of $A_{t}$ depends upon $t$ in the following sense:

(T) If $\beta>0, t_{n} \uparrow t$ in $(0, T], u_{n} \in X_{\beta, t_{n}}$ and $u_{n} \rightarrow u$ in $X$, then $u \in \bar{X}_{a, t}$ for some $\alpha$ which is $\geqq \beta$ and depends only upon $\beta$.

Next, using the class $\mathscr{I}$ and taking a subset $C$ of $X$, we consider the following condition:

$(\boldsymbol{H} ; C)$ There exists a number $\omega \geqq 0$ and a function $\theta \in \mathscr{I}$ such that

$$
\tau_{-}(v, u-x)+\tau_{-}(-y, u-x) \leqq \omega\|u-x\|+\theta(t, s)
$$

holds for $s, t \in[0, T],(u, v) \in A_{t}$ and $(x, y) \in A_{s}$ with $u, x \in C$.

In this condition $\omega$ and $\theta$ may depend upon the choice of $C$. Since $\theta(t, t)=0$, condition $(\boldsymbol{H} ; C)$ implies that each $A_{t}$ is $\omega$-quasi-dissipative on $C$ in the sense of Kobayashi [13].

In this paper we specify the subset $C$ that appears in condition $(\boldsymbol{H} ; C)$ by means of an 1.s.c. functional $\mathfrak{p}$ on $X$ and employ conditions $\left(\boldsymbol{H} ; X_{\omega}\right), \alpha>0$. (It turns out that $\mathfrak{p}$ specifies the class of operators $A_{t}$ as well as the $t$-dependence of $A_{t}$.)

We have thus introduced three types of conditions $(\boldsymbol{P}),(\boldsymbol{T})$ and $\left(\boldsymbol{H} ; X_{\omega}\right)$. We are mainly concerned with solutions of evolution equations $(D E)_{s}$ with initial-data belonging to the sets $\bar{X}_{\beta, s}(\beta>0)$, and we shall put the following condition as our basic hypothesis on the family of operators $\left\{A_{t}\right\}$ :

Hypothesis $(\boldsymbol{H})$. There is an 1.s.c. functional $\mathfrak{p}: X \rightarrow[0, \infty]$ satisfying $(\boldsymbol{P})$ and $(\boldsymbol{T})$; and for every $\alpha>0$, the family $\left\{A_{t}\right\}$ satisfies $\left(\boldsymbol{H} ; X_{\boldsymbol{\omega}}\right)$ for some $\omega_{\infty} \geqq 0$ and $\theta_{\infty} \in \mathcal{I}$.

\section{Remarks on the basic hypothesis $(H)$.}

The $t$-dependence is usually discussed in connection with initial-data though we shall treat initial-data belonging to $X_{\beta} \cap \overline{D\left(A_{s}\right)}$ and construct the associated solutions of $(D E)_{s}$ in some $X_{a}$ (depending possibly upon the choice of $\beta$ ). It seems to be too restricted to assume the existence of the function $\theta$ mentioned as in $\left(\boldsymbol{H} ; X_{\sigma}\right)$ since it asserts the uniform $t$-dependence of $A_{t}$ on the closed set $X_{\omega}$, though this assumption is fairly general. To see this, we here state the relation of hypothesis $(\boldsymbol{H})$ to the conditions that were treated in earlier works quoted in the Introduction.

REMARK 1.1. In many cases (and for all the applications of our results mentioned as in [9], [15], [16] and [23]) the following stronger condition is 
employed, instead of $(\boldsymbol{H} ; \boldsymbol{C})$.

$(\boldsymbol{H} ; C)^{\prime} \quad$ There exist $\omega \geqq 0$ and $\theta \in \mathscr{I}$ such that

$$
\tau_{-}(v-y, u-x) \leqq \omega\|u-x\|+\theta(t, s)
$$

for $s, t \in[0, T],(u, v) \in A_{t} / C$ and $(x, y) \in A_{s} / C$. Here $A / C$ denotes the restriction of $A$ to a set $C$. Condition $(\boldsymbol{H} ; C)^{\prime}$ states that $A_{t}-\omega$ is dissipative on $C$ for each $t \in[0, T]$. Hence $I-\lambda\left(A_{t} / C\right)$ is injective for $\lambda \in(0,1 / \omega)$ and $\left(I-\lambda\left(A_{t} / C\right)\right)^{-1}$ is Lipschitz continuous with Lipschitz constant $(1-\omega \lambda)^{-1}$. See [2] and [7]. Hereafter we say that $A_{t}$ is $\omega$-dissipative on $C$ if $A_{t}-\omega$ is dissipative on $C$; if in particular $C=X$, we say simply that $A_{t}$ is $\omega$-dissipative. Condition $\left(\boldsymbol{H} ; X_{\boldsymbol{\omega}}\right)^{\prime}$ implies $\left(\boldsymbol{H} ; X_{\boldsymbol{\omega}}\right)$. But it is not hard to find examples of quasi-dissipative operators which are not dissipative.

REMARK 1.2. If $\mathfrak{p}(x) \equiv 0$ on $X$ (hence $X_{\omega}=X$ for $\alpha>0$ ) and $\omega=0$ in $(\boldsymbol{H} ; X)$, then each of $A_{t}$ is quasi-dissipative in the sense of Takahashi [30]. Suppose $\mathfrak{p}(\cdot)$ is defined to be the norm $\|\cdot\|$ of $X$. Then $\left(\boldsymbol{H} ; X_{\boldsymbol{\omega}}\right)$ states that there is a number $\omega_{\infty} \geqq 0$ and $A_{t}$ is $\omega_{\infty}$-quasi-dissipative on the closed ball $X_{\omega}$ with center 0 and radius $\alpha$. This is the same setting as in Chambers-Oharu [3] and it turns out that our argument treats the evolution governed by more general operators than dissipative operators. From the viewpoint of the theory of ordinary differential equations, it is more adequate to assume that the inequality $\tau_{-}(v-y, u-x) \leqq \omega(\|u-x\|)+\theta_{\omega}(t, s)$ holds for $(u, v) \in A_{t},(x, y) \in A_{s}$ with $u, x \in X_{\infty}$ and some function $\omega:[0, \infty) \rightarrow[0, \infty)$ with $\omega(0)=0$. But this condition is a special case of Hypothesis $(\boldsymbol{H})$ provided that $p(\cdot)=\|\cdot\|$ on $X$ and $\omega \in \mathcal{C}^{1}[0, \infty)$.

REMARK 1.3. As mentioned in Section 0, our hypothesis $(\boldsymbol{H})$ does not necessarily extend all of the conditions treated by Crandall-Pazy [4] and Evans [8]. In order to cover them we need another type of hypothesis. Taking two subset $C$ and $C^{\prime}$ of $X$, we consider the following condition:

$\left(\boldsymbol{H}^{*} ; C, C^{\prime}\right)$ There exist $\omega \geqq 0, \lambda_{0}>0$ and $\theta \in \mathcal{I}$ such that $A_{t}$ is $\omega$-dissipative on $C$ for $t \in[0, T]$; and if $(u, v) \in A_{t},(x, y) \in A_{s}, u, x \in C, w=u-\lambda v \in C^{\prime}$ and $z=x-\lambda y \in C^{\prime}$ for $s, t \in[0, T]$, then we have

$$
(1-\omega \lambda)\|u-x\| \leqq\|w-z\|+\lambda \theta(t, s) \text { for } 0<\lambda<\lambda_{0}(\leqq 1 / \omega) .
$$

In this condition $\omega$ depends possibly on the choice of $C$, while $\lambda_{0}$ and $\theta$ may depend upon the choice of $C^{\prime}$ as well as $C$. It should be noted that $\left(H^{*} ; C, C^{\prime}\right)$ does not make sense unless $R\left(I-\lambda A_{t} / C\right)$ intersects $C^{\prime}$ for $t \in[0, T]$ and $\lambda \in\left(0, \lambda_{0}\right)$. Condition $\left(\boldsymbol{H}_{2} ; C, X\right)$ always makes sense and is stronger than $\left(\boldsymbol{H}_{2} ; C, C^{\prime}\right)$ provided that $R\left(I-\lambda A_{t} / C\right)$ intersects $C^{\prime}$ for $t \in[0, T]$ and $\lambda \in\left(0, \lambda_{0}\right)$. No general relationships between conditions $(\boldsymbol{H} ; C)$ and $\left(\boldsymbol{H}^{*} ; C, C^{\prime}\right)$ 
can be obtained since these conditions are quite involved. Now Conditions $\left(\boldsymbol{H}^{*} ; X_{\omega}, X_{\beta}\right)$ are related to the $t$-dependence of $A_{t}$ discussed in [4], [5] and [8]. For the detailed argument, see the forthcoming paper [18].

REMARK 1.4. After we had obtained some of the main results (that are announced in Kobayashi [14]), we received a preprint of the recent work of Pavel [25]. His work is closely related to our paper since he discussed the construction of evolution operators associated with the system $\left\{(D E)_{s}\right\}$ for $\omega$-quasi-dissipative operators through the same type of discrete approximation as mentioned in Section 0 . The $t$-dependence of the operator $A_{t}$ with which he is concerned is that of the form $\left(\boldsymbol{H} ; X_{\alpha}\right)$. More precisely, if $p$ is the norm of $X, \omega$ a nonnegative constant, and if $\theta_{\alpha}(t, s)=\|\boldsymbol{a}(t)-\boldsymbol{a}(s)\| L(\alpha)$ for $\alpha>0$ and some $\boldsymbol{a}(\cdot) \in \boldsymbol{L}^{1}(0, T ; X)$, then the family $\left\{A_{t}\right\}$ is $\boldsymbol{a}(\cdot)$ - $\omega$-quasi-dissipative in the sense of Definition 2.1 of [25] iff $\left\{A_{t}\right\}$ satisfies condition $\left(\boldsymbol{H} ; X_{a}\right)$ for the $\mathfrak{p}, \omega, \theta_{\boldsymbol{\alpha}}$, and $\alpha>0$. Therefore, his convergence argument for the discrete approximation may be treated within the framework of our discussion through an suitable approximation for the Bochner integrable function $\boldsymbol{a}(\cdot)$. In fact, it is possible to extend our results below to more general ones so that the above cases can be directly treated, though we do not go into such generalizations in this paper because of considerable complexity.

\section{Generalized solutions}

In this section we introduce two notions of generalized solutions to the IVP for $(D E)_{s}$ and discuss basic properties of the generalized solutions as well as the relationships between them. As the main result of this section, a uniqueness theorem for the weak solution is established.

Throughout this section we put Hypothesis $(\boldsymbol{H})$. Since the parameter $s$ in equation $(D E)_{s}$ plays no essential role in this section, we restrict ourselves to the single equation $(D E)_{0}$ (hereafter denoted $(D E)$ ) with initial-condition $(I C)_{0}$ (likewise, denoted $(I C)$ ).

We use five spaces of vector-valued functions: Given a bounded half-open interval $\boldsymbol{J}=[a, b), B(\boldsymbol{J} ; X)$ denotes the space of $X$-valued, uniformly bounded functions on $J$ endowed with the systems of countable seminorms defined by

$$
\|\| u \|_{n}=\sup \{\|u(t)\|: a \leqq t \leqq b-1 / n\}, n>[b-a] ;
$$

and $\boldsymbol{C}(\boldsymbol{J} ; X)$ denotes the subspace of $\boldsymbol{H}(\boldsymbol{J} ; X)$ equipped with the same seminorm system. While, given a compact interval $K=[a, b], \boldsymbol{B}(K ; X)$ denotes the Banach space of $X$-valued, uniformly bounded functions on $\boldsymbol{J}$ endowed with the usual supremum norm; $\boldsymbol{C}(K ; X)$ denotes the closed subspace of $\boldsymbol{B}(K ; X)$ consisting of strongly continuous functions; and $\boldsymbol{L}^{1}(K ; X)$ denotes the usual Lebesgue space of $X$-valued, Bochner integrable functions on $K$ endowed with the norm 
$\|u\|_{\mathbf{L}^{1}(K ; X)}=\int_{K}\|u(t)\| d \mu$.

2.1. Weak solutions.

We begin by introducing a notion of approximate solution to equation $(D E)$. Let $\varepsilon>0$. An $X$-valued function, say $u_{\mathrm{g}}$, in $\boldsymbol{C}([0, T] ; X)$ is called an E-approximate solution to $(D E)$ if there exists a partition $\Delta_{\mathrm{g}}=\left\{0=t_{0}<t_{1}<\cdots\right.$ $\left.<t_{N} \leqq t_{N+1}=T\right\}$ of $[0, T]$ and an $X$-valued step function $f_{\mathrm{z}}=\sum_{K=1}^{N} f_{k}^{\mathbb{Q}} \chi_{\left(t_{k-1}, t_{k}\right]}$ in $L^{1}(0, T ; X)$ satisfying two conditions below:

(a $\left.a_{1}\right) \quad\left|\Delta_{\varepsilon}\right|=\max \left\{\left|t_{k}-t_{k-1}\right|: 1 \leqq k \leqq N+1\right\}<\varepsilon$ and $\left\|f_{\varepsilon}\right\|_{L^{1}(0, T ; x)}<\varepsilon$.

$\left(\mathrm{a}_{2}\right) \quad$ For each $k \in\{1,2, \cdots, N\}, u_{\varepsilon}\left(t_{k}\right) \in D\left(A_{t_{k}}\right)$ and

(*) $\frac{u_{\mathrm{g}}\left(t_{k}\right)-u_{\mathrm{g}}\left(t_{k-1}\right)}{t_{k}-t_{k-1}}-f_{\mathrm{g}}\left(t_{k}\right) \in A_{t_{k}} u_{\mathrm{g}}\left(t_{k}\right)$.

The system of equations $(*)$ displayed above is regarded as an approximate scheme for $(D E)$ and $f_{\varepsilon}$ is understood to mean an error function. Accordingly, if there exists a sequence $\left\{u_{\varepsilon_{n}}\right\}$ of $\varepsilon_{n}$-approximate solutions and a function $u$ in $\mathcal{C}([0, T) ; X)$ such that $u_{\varepsilon_{n}}$ converges in $\mathcal{C}([0, T) ; X)$ to $u$ as $n \rightarrow \infty$, it is expected that $u$ is a solution of $(D E)$ (perhaps in a generalized sense). We then give a notion of weak solution through the $\varepsilon$-approximate solutions.

Definition 2.1. Let $\alpha>0$. An $X$-valued function $u$ in $\mathcal{C}([0, T] ; X)$ is said to be a weak solution of $(D E)$ constrained in $X_{\infty}$, if there exists a sequence $\left\{u_{n}\right\}$ of $\varepsilon_{n}$-approximate solutions satisfying:

$\left(w_{1}\right) \quad$ For every $n \geqq 1$, let $\Delta_{n}=\left\{0=t_{0}^{n}<t_{1}^{n}<\cdots<t_{N_{n}}^{n} \leqq t_{N_{n}+1}^{n} \equiv T\right\}$

and $f_{n} \in L^{1}(0, T ; X)$ be respectively the partition of $[0, T]$ and the error function associated with the $\varepsilon_{n}$-approximate solution $u_{n}$. Let $h_{k}^{n}=t_{k}^{n}-t_{k-1}^{n}$ for $1 \leqq k \leqq N_{n}$. Then for every $k \in\left\{1,2, \cdots, N_{n}\right\}, u_{n}\left(t_{k}^{n}\right) \in D\left(A_{t_{k}^{n}}\right) \cap X_{\alpha}$ and $\left(h_{k}^{n}\right)^{-1}\left[u_{n}\left(t_{k}^{n}\right)-\right.$ $\left.u_{n}\left(t_{k-1}^{n}\right)\right]-f_{n}\left(t_{k}^{n}\right) \in A_{t_{k}^{n}} u_{n}\left(t_{k}^{n}\right)$.

$\left(w_{2}\right) \quad \lim _{n \rightarrow \infty} \varepsilon_{n}=0, \quad \lim _{n \rightarrow \infty}\left|\Delta_{n}\right|=0, \quad$ and $\quad \lim _{n \rightarrow \infty}\left\|f_{n}\right\|_{L^{1}(0, T ; X)}=0$.

$\left(w_{3}\right) \quad u_{n}$ converges in $\mathcal{C}([0, T) ; X)$ to $u$ as $n \rightarrow \infty$.

(Therefore, the range of $u$ is contained in the closed set $X_{\infty}$ ).

Interpretations of the above definition from the point of view of the difference approximation for $(D E)$ as well as that of the strong solution to $(D E)$ are in order.

Given $\beta>0$ and $x \in \bar{X}_{\beta, 0}=\overline{X_{\beta} \cap D\left(A_{0}\right)}$, suppose that there exists a number $\alpha \geqq \beta$ and a system $\left(\left\{\Delta_{n}\right\},\left\{\left(u_{k}^{n}, v_{k}^{n}\right)\right\},\left\{f_{k}^{n}\right\}\right)$ of a number and sequences with the properties (i), (ii) as mentioned in Section 0 and

(iii) $\left\{\left(u_{k}^{n}, v_{k}^{n}\right)\right\}$ is a sequence of elements in $X_{\alpha} \times X$. 
In what follows, we denote such scheme by $((D S))$ which, in Section 0 , we called a consistent discrete scheme for $(D E)$. We then define step functions $u^{n}:[0, T] \rightarrow X(n \geqq 1)$ by the equations $u^{n}(0)=u_{0}^{u}$ and

$$
u^{n}(t)=\sum_{k=1}^{N_{n}} u_{k}^{n} \chi_{\left(t_{k-1}^{n}, t_{k}^{n}\right]}(t) \text { for } t \in(0, T] .
$$

The sequence $\left\{u^{n}\right\}$ of step functions may also be regarded as a sequence of approximate solutions to $(D E)$. Hence, if there is an $X$-valued function $u$ in $\boldsymbol{C}([0, T], X)$ and the convergence

$$
u(t)=\lim _{n \rightarrow \infty} u^{n}(t)
$$

holds in $\boldsymbol{B}([0, T), X)$, it is also expected that $u$ is a solution (in a generalized sense) of $(D E)$. Following Kobayashi [13], the limit function $u$ may be called a $D S$-limit solution with initial-value $x$ of $(D E)$ constrained in $X_{*}$. Now the relation of the $D S$-limit solution of $(D E)$ to the weak solution can be stated as follows.

Proposition 2.1. A function $u$ in $\boldsymbol{C}([0, T] ; X)$ with $u(0)=x \in X_{a}$ is a weak solution of $(D E)$ constrained in $X_{\infty}$ iff it is a DS-limit solution with initial-value $x$ of $(D E)$ constrained in $X_{\text {w }}$.

Proof. First suppose that (2.1) holds for $u$ and $a$ consistent discrete scheme $\left(\left\{\Delta_{n}\right\},\left\{\left(u_{k}^{n}, v_{k}^{n}\right)\right\},\left\{f_{k}^{n}\right\}\right)$ with the properties (i), (ii) and (iii) as mentioned above. Then a sequence $\left\{u_{n}\right\}$ of piecewise linear functions in $\boldsymbol{C}([0, T] ; X)$ can be constructed by connecting the points $u_{k}^{n}\left(0 \leqq k \leqq N_{n}\right)$ with line sequents successively. The sequence $\left\{u_{n}\right\}$ gives a sequence of $\left|\Delta_{n}\right|$-approximate solutions with properties $\left(w_{1}\right)-\left(w_{3}\right)$ as mentioned in Definition 2.1 and the strong continuity on $[0, T]$ of $u$ (which follows from (2.1) and will be shown in Theorem 2.4 below) implies that $u_{n} \rightarrow u$ in $\mathcal{C}([0, T) ; X)$. Conversely, let $u$ be a weak solution of $(D E)$ constrained in $X_{\infty}$ and $\left\{u_{n}\right\}$ a sequence of $\varepsilon_{n}$-approximate solutions satisfying $\left(w_{1}\right)-\left(w_{3}\right)$. If we define $u_{k}^{n}=u_{n}\left(t_{k}^{n}\right), f_{k}^{n}=f_{n}\left(t_{k}^{n}\right)$ and $v_{k}^{n}=\left(h_{k}^{n}\right)^{-1}\left[u_{k}^{n}-u_{k-1}^{n}\right]-$ $f_{k}^{n}$ for $n \geqq 1$ and $1 \leqq k \leqq N_{n}$, then the system $\left(\left\{\Delta_{n}\right\},\left\{\left(u_{k}^{n}, v_{k}^{n}\right)\right\},\left\{f_{k}^{n}\right\}\right)$ forms a consistent descrete scheme for $(D E)_{s}$ in $X_{\alpha}$. Moreover, (2.1) holds for $u$ since $u \in \mathcal{C}([0, T] ; X)$.

q.e.d.

Moreover, the class of all weak solutions is a family in $\mathcal{C}([0, T] ; X)$ such that no strong solutions of $(D E)$ can exist outside the family, as'mentioned below.

Proposition 2.2. Suppose that there is a function $u:[0, T] \rightarrow X$ such that $u([0, T]) \subset X_{\text {os }}$ for some $\alpha>0$, and that $u$ is Lipschitz continuous on $[0, T]$, strongly differentiable at a.e. $t \in[0, T]$, and satisfies $(D E)$ for a.e. $\imath \in[0, T]$. Then, there is a system $\left(\left\{\Delta_{n}\right\},\left\{\left(u_{k}^{n}, v_{k}^{n}\right)\right\},\left\{f_{k}^{n}\right\}\right)$ satisfying (i), (ii) and (iii); and the convergence 
(2.1) holds in $B([0, T] ; X)$.

Proof. We mimic the proof of Kenmochi-Oharu [12], Theorem 4.12. Let $v(t)=u^{\prime}(t)$ and let $N$ be a Lebesgue null set such that $v(t) \in A_{t} u(t)$ for $t$ $\mathrm{t} \in[0, T]-N$. Let $\left\{\delta_{m}\right\}$ be any null sequence in $(0, T)$ and set $w_{m}(t)=$ $\delta_{m}^{-1}\left[u(t)-u\left(t-\delta_{m}\right)\right]$ if $t \in\left[\delta_{m}, T\right]$ and $w_{m}(t)=0$ if $t \in\left[0, \delta_{m}\right)$. Since $w_{m}(t) \rightarrow v(t)$ for $t \in[0, T]-N$, Egoroff's theorem yields that for each $n \geqq 1$ there is a closed subset $E_{n}$ of $(0, T)$ such that $\mu\left([0, T]-E_{n}\right)<1 / n, E_{n} \cap N=\emptyset$, and $\sup \left\{\left\|w_{m}(t)-v(t)\right\|: t \in E_{n}\right\} \rightarrow 0$ as $m \rightarrow \infty$, where $\mu$ denotes the Lebesgue measure restricted on $[0, T]$. Fix any $n \geqq 1$ and let $m$ be such that

$$
0<\delta_{m}<1 / n \text { and }\left\|w_{m}(t)-v(t)\right\| \leqq 1 / n \text { for } t \in E_{n} .
$$

Now set $u_{0}^{n}=u(0)$ and $t_{0}^{n}=0$. We shall construct finite sequences $\left\{\nu_{k}^{n}\right\}_{k=1}^{N_{n}}$, $\left\{t_{k}^{n}\right\}_{k=1}^{N_{n}}$ and $\left\{\left(u_{k}^{n}, v_{k}^{n}\right)\right\}_{k=1}^{N_{n}}$ with the properties as mentioned in (2.3) below. In what follows, we eliminate for simplicity in notation the suffix $n$ from the symbols $\nu, N, t, u, v$ and $E$ since it will cause no confusion:

$$
\begin{aligned}
& t_{k}=t_{k-1}+\delta_{m}+\nu_{k} \in E, \quad \nu_{k} \geqq 0, \quad \sum_{k=1}^{N} \nu_{k} \leqq 1 / n, \quad u_{k} \in X, \\
& \left(u_{k}, v_{k}\right) \in A_{t_{k}} \text { and }\left\|u_{k}-\left(\delta_{m}+\nu_{k}\right) v_{k}-u_{k-1}\right\| \leqq \delta_{m} / n+2 L \nu_{k},
\end{aligned}
$$

where $1 \leqq k \leqq N, \lim _{n \rightarrow \infty} t_{N}=T$ and $L$ denotes the smallest Lipschitz constant for the solution $u$. First define $\nu_{1}=\operatorname{dist}\left(\delta_{m}, E-\left[0, \delta_{m}\right)\right)$. Since $E-\left[0, \delta_{m}\right)$ is closed, we infer that $t_{1}=t_{0}+\delta_{m}+\nu_{1} \in E$ and $\left(\delta_{m}, \delta_{m}+\nu_{1}\right) \cap E=\emptyset$. We then set $u_{1}=u\left(t_{1}\right)$ and $v_{1}=v\left(t_{1}\right)$. By (2.2) and the Lipschitz continuity of $u(\cdot)$ we have $\left\|u_{1}-\left(\delta_{m}+\nu_{1}\right) v_{1}-u_{0}\right\| \leqq\left\|u\left(t_{1}\right)-\delta_{m} v\left(t_{1}\right)-u\left(t_{0}+\nu_{1}\right)\right\|+\nu_{1}\left\|v\left(t_{1}\right)\right\|+\left\|u\left(t_{0}+\nu_{1}\right)-u\left(t_{0}\right)\right\| \leqq$ $\delta_{m} / n+2 L \nu_{1}$. Moreover, it is clear that $u_{1} \in X_{\infty}$ and $\left(u_{1}, v_{1}\right) \in A_{t_{1}}$. This completes the first stage of our construction. Next, suppose that the $k$-th step of the construction has been completed: hence we have finite sequences $\nu_{l}=$ $\operatorname{dist}\left(t_{l-1}+\delta_{m}, E-\left[0, t_{l-1}+\delta_{m}\right)\right), t_{l}=t_{l-1}+\delta_{m}+\nu_{l}, u_{l}=u\left(t_{l}\right)$, and $v_{l}=v\left(t_{l}\right)$, $l=1,2, \cdots, k$, with the property that $t_{l} \in E,\left(t_{l-1}+\delta_{m}, t_{l}\right) \cap E=\emptyset, u_{l} \in X_{w}$, $\left(u_{l}, v_{l}\right) \in A_{t_{l}}$ and $\left\|u_{l}-\left(\delta_{m}+\nu_{l}\right) v_{l}-u_{l-1}\right\| \leqq \delta_{m} / n+2 L \nu_{l}$ We perform the $(t+1)^{\text {th }}$ step in the following way: If $\left(t_{k}+\delta_{m}, T\right] \cap E=\emptyset$, we set $k=N$ and finish our construction since $T-t_{k}<1 / n$. If $\left(t_{k}+\delta_{m}, T\right] \cap E \neq \emptyset$, then set $\nu_{k+1}=$ $\operatorname{dist}\left(t_{k}+\delta_{m}, E-\left[0, t_{k}+\delta_{m}\right)\right)$ and define $t_{k+1}=t_{k}+\delta_{m}+\nu_{k+1}, u_{k+1}=u\left(t_{k+1}\right)$ and $v_{k+1}=v\left(t_{k+1}\right)$. Then $t_{k+1} \in E$ by the closedness of $E-\left[0, t_{k}+\delta_{m}\right)$, so that $u_{k+1} \in X_{o s}$, $\left(u_{k+1}, v_{k+1}\right) \in A_{t_{k+1}}$ and we have $\left\|u_{k+1}-\left(\delta_{m}+\nu_{k+1}\right) v_{k+1}-u_{k}\right\| \leqq \| u\left(t_{k+1}\right)-\delta_{m} v\left(t_{k+1}\right)-$ $u\left(t_{k}+\nu_{k+1}\right)\left\|+\nu_{k+1}\right\| v\left(t_{k+1}\right)\|+\| u\left(t_{k}+\nu_{k+1}\right)-u\left(t_{k}\right) \| \leqq \delta_{m} / n+2 L \nu_{k+1}$. Moreover, $\left(t_{k}+\delta_{m}, t_{k+1}\right) \cap E=\emptyset, u_{k} \in X_{\infty}$ and $\left(u_{k+1}, v_{k+1}\right) \in A_{t_{k+1}}$ by the same reason as above. Repeating this argument, we obtain finite sequences $\left\{\nu_{k}\right\},\left\{t_{k}\right\}$ and $\left\{\left(u_{k}, v_{k}\right)\right\}$ satisfying (2.3).

Now let $h_{k}=\delta_{m}+\nu_{k}$ and $f_{k}=\left(h_{k}\right)^{-1}\left[u_{k}-u_{k-1}\right]-v_{k}$ for $1 \leqq k \leqq N$. Then $h_{k}=t_{k}-t_{k-1}$ and $h_{k}\left\|f_{k}\right\|=\left\|u_{k}-h_{k} v_{k}-u_{k-1}\right\| \leqq \delta_{m} / n+2 L \nu_{k}$ for $1 \leqq k \leqq N$, so that if 
we define $\Delta=\left\{0=t_{0}<t_{1}<\cdots<t_{N} \leqq t_{N+1}=T\right\}$ then $|\Delta|=\max \left\{\delta_{m}+\nu_{k}: 1 \leqq k \leqq N\right\}$ $\leqq 2 / n, \sum_{k=1}^{N} h_{k}\left\|f_{k}\right\| \leqq T / n+2 L \sum_{k=1}^{N} \nu_{k} \leqq(T+2 L) / n$ and $T-t_{N}<1 / n$. Denoting $N, \Delta, \nu_{k}, t_{k}, u_{k}$ and $v_{k}$ constructed in the above manner, respectively, by $N_{n}$, $\Delta_{n}, v_{k}^{n}, t_{k}^{n}, u_{k}^{n}$ and $v_{k}^{n}$, we obtain the desired system $\left(\left\{\Delta_{n}\right\},\left\{\left(u_{k}^{n}, v_{k}^{n}\right)\right\},\left\{f_{k}^{n}\right\}\right)$.

q.e.d.

The function $u$ mentioned as in Proposition 2.2 is called a strong solution of $(D E)$. In view of Lemma 1.1 of Kato [11], such a solution is unique if each $A_{t}$ is $\omega_{a}$-dissipative on $X_{a}$ for some $\omega_{a} \geqq 0$. Moreover the proof of Proposition 2.2 shows that a strong solution itself is an $\varepsilon$-approximate solution for every $\varepsilon>0$ and $a$ fortiori a weak solution.

\subsection{Integral solutions.}

The notion of weak solution to $(D E)$ is defined under the assumption that a sequence of $\varepsilon_{n}$-approximate solutions or else a consistent discrete scheme $((D S))$ for $(D E)$ exists. Here we introduce another notion of generalized solution.

Suppose for the moment that a function $u:[0, T] \rightarrow X_{\infty}$ is a strong solution of $(D E)$. Let $\gamma \geqq \alpha$. Since $u(t) \in X_{\gamma}$ for $t \in[0, T]$ and $u^{\prime}(t) \in A_{t} u(t)$ for a.e. $t \in[0, T]$, the application of Lemma 1.1 of Kato [11] and condition $\left(H ; X_{\gamma}\right)$ yields $(d / d t)\|u(t)-x\|=\tau_{-}\left(u^{\prime}(t), u(t)-x\right) \leqq \tau_{+}(y, u(t)-x)+\omega\|u(t)-x\|+\theta(t, r)$ for a.e. $t$, where $r \in[0, T],(x, y) \in A_{\gamma}, x \in X_{\gamma}$; and $\omega, \theta$ are specified by condition $\left(H ; X_{\gamma}\right)$. Notice that the right side of the above inequality is bounded and summable over $[0, T]$. Integrating both sides over any subinterval $\left[t, t^{\prime}\right]$ of $[0, T]$ yields

$$
\left\|u\left(t^{\prime}\right)-x\right\|-\|u(t)-x\| \leqq \int_{t}^{t^{\prime}}\left[\tau_{+}(y, u(\xi)-x)+\omega\|u(\xi)-x\|+\theta(\xi, r)\right] d \xi
$$

The above observation was motivated by Bénilan's idea. Although (2.4) does not seem to give a generalized form of equation $(D E)$, the integral inequality (2.4) plays an important role in the discussion of generalized solutions to $(D E)$. Let $u \in C\left([0, T] ; X_{\alpha}\right)$ and suppose that $u(t) \in \bar{X}_{\alpha, t}$ for $t \in[0, T]$ and (2.4) (with $\gamma=\alpha$ ) holds for $0 \leqq t \leqq t^{\prime} \leqq T, r \in[0, T]$ and $(x, y) \in A_{r}$ with $x \in X_{\alpha}$. If $u$ is weakly right-differentiable at $t_{0} \in(0, T)$ and $A_{t_{0}}-\omega$ is maximal dissipative on $\bar{X}_{a, t_{0}}$, then $u\left(t_{0}\right) \in D\left(A_{t_{0}}\right)$ and $\left(w-d^{+} / d t\right) u\left(t_{0}\right) \in A_{t_{0}} u\left(t_{0}\right)$, where $\left(w-d^{+} / d t\right) u\left(t_{0}\right)$ denotes the weak right-derivative of $u$ at $t_{0}$. In fact, given $(x, y) \in A_{t_{0}} / X_{\infty}$ and $h \in\left(0, T-t_{0}\right)$, we have

$$
\begin{gathered}
\tau_{+}\left(h^{-1}\left[u\left(t_{0}+h\right)-u\left(t_{0}\right)\right], \quad u\left(t_{0}\right)-x\right) \leqq h^{-1}\left(\left\|u\left(t_{0}+h\right)-x\right\|-\left\|u\left(t_{0}\right)-x\right\|\right) \\
\leqq h^{-1} \int_{t_{0}}^{t_{0}+h}\left[\tau_{+}(y, u(\xi)-x)+\omega\|(\xi)-x\|+\theta\left(\xi, t_{0}\right)\right] d \xi .
\end{gathered}
$$

Letting $h \downarrow 0$ and applying the relation $\tau_{-}(v-w, u) \leqq \tau_{+}(v, u)-\tau_{+}(w, u)$, we get 
$\tau_{-}\left(\left(w-d^{+} / d t\right) u\left(t_{0}\right)-y, u\left(t_{0}\right)-x\right) \leqq \omega\left\|u\left(t_{0}\right)-x\right\|$. The maximal dissipativity of $A_{t_{0}}-\omega$ on $\bar{X}_{a, t_{0}}$ then implies that $\left(u\left(t_{0}\right),\left(w-d^{+} / d t\right) u\left(t_{0}\right)\right) \in A_{t_{0}}$. In particular, we have:

Proposition 2.3. Suppose that $A_{t}-\omega$ is maximal dissipative on $\bar{X}_{a, t}$ for $t \in[0, T]$. Let $u:[0, T] \rightarrow X_{\infty}$ be specified as above. If $u$ is weakly differentiable a.e. on $[0, T]$, then $u$ satisfies the equation

$$
\left.(w-d / d t) u(t) \in A_{t} u(t) \quad \text { for a.e. } t \in 0, T\right) \text {. }
$$

Now the above observations lead us to the:

Definition 2.2. Let $\alpha>0$ and $s \in[0, T)$. An $X$-valued, strongly continuous function $u$ on $[s, T]$ is said to be an integral solution of $(D E)_{s}$ constrained in $\left\{\bar{X}_{\omega, t}: t \in[s, T]\right\}$ if $u(t) \in \bar{X}_{\alpha, t}$ for $t \in[s, T]$ and there exist $\omega \geqq 0$ and $\theta \in \mathcal{I}$ for which the integral inequality (2.4) holds for $r \in[0, T],(x, y) \in A_{r}$ with $x \in X_{\infty}$ and $0 \leqq t \leqq t^{\prime} \leqq T$. (Note that the function $\tau_{+}(y, u(\cdot)-x)$ is bounded and upper simicontinuous on $[0, T]$.)

Hereafter we denote by $\mathcal{I}_{a}[0, T]$ the class of all integral solutions of $(D E)$ constrained in $\left\{\bar{X}_{\alpha, t}\right\}$. When we are concerned with equation $(D E)_{s}(s \in(0, T))$, the class of all integral solutions of $(D E)_{s}$ constrained in $\left\{\bar{X}_{a, t}: s \leqq t \leqq T\right\}$ is denoted by $\mathcal{G}_{a}[s, T]$.

The class of Bénilan's integral solutions introduced in [1] does not necessarily specify a proper class of solutions (since the class is in general too large to be a reasonable class of generalized solutions), but rather specify a class of $X$-valued functions which contains the generalized solutions in a very natural way. Now the class $\mathcal{I}_{\alpha}[0, T]$ is closed under the uniform convergence, and $\mathcal{G}_{\infty}[0, T]$ may also be too large to be a class of generalized solutions to $(D E)$. However, Definition 2.2 is a straightforward extension of Benilan's integral solution to our time-dependent case and we have the following result which states that a weak solution of $(D E)$ satisfying a given initial condition $(I C)$ is uniquely determined in the classes $\mathcal{G}_{\gamma}[0, T]$.

Theorem 2.4. Let $\beta>0$ and $x \in \bar{X}_{\beta, 0}=\overline{X_{\beta} \cap D\left(A_{0}\right)}$. Suppose that a consistent discrete scheme $((D S))$ for $(D E)$ and $(I C)$ exists in some $X_{\alpha}(\alpha \geqq \beta)$, and that the convergence (2.1) holds in $B([0, T), X)$. Then, the limit function $u$ of (2.1) gives an iniegral solution of $(D E)$ constrained in $\left\{\bar{X}_{\gamma, t}\right\}$ for some $\gamma \geqq \alpha$. Moreover, for every $\gamma^{\prime} \geqq \gamma$ the limit function $u$ is the oniy element of $\mathcal{G}_{\gamma^{\prime}}[0, T]$ satisfying $u(0)=x$.

Proof. We first use Lemma 3.4 (mentioned later) to show that the limit function $u$ of $(2.1)$ can be extended to a strongly continuous function on $[0, T]$. Let $\left(\left\{\Delta_{m}\right\},\left\{\left(u_{j}^{m}, v_{j}^{m}\right)\right\},\left\{f_{j}^{m}\right\}\right)$ be the consistent discrete scheme $((D S))$ for $(D E)$ 
in $X_{\alpha}$. Let $s=0,(\tilde{x}, \tilde{y}) \in A_{0}, \tilde{x} \in X_{\beta}, \hat{t}_{j}^{m}=t_{j}^{m}, \hat{u}_{j}^{m}=u_{j}^{m}, \hat{h}_{j}^{m}=h_{j}^{m}, \hat{\Delta}_{m}=\Delta_{m}$ and $\hat{x}=x$ in (3.9). Then the application of Lemma 3.4 yields the estimate

$$
\begin{aligned}
& \prod_{p=1}^{k}\left(1-\omega h_{p}^{n}\right) \prod_{q=1}^{j}\left(1-\omega h_{q}^{m}\right)\left\|u^{n}\left(t_{k}^{n}\right)-u^{m}\left(t_{j}^{m}\right)\right\| \\
\leqq & \left\|u_{0}^{n}-\tilde{x}\right\|+\left\|\tilde{x}-u_{0}^{m}\right\|+\sum_{p=1}^{N} h_{p}^{n}\left\|f_{p}^{n}\right\|+\sum_{q=1}^{N} h_{q}^{m}\left\|f_{q}^{m}\right\| \\
+ & {\left[\left(t_{k}^{n}-t_{j}^{m}\right)^{2}+\left|\Delta_{n}\right| t_{k}^{n}+\left|\Delta_{m}\right| t_{j}^{m}\right]^{1 / 2}(\|\tilde{y}\|+\rho(T)) } \\
+ & t_{j}^{m} \varepsilon^{-1} \rho(T)\left[\left(t_{k}^{n}-t_{j}^{m}-\eta\right)^{2}+\left|\Delta_{n}\right| t_{k}^{n}+\left|\Delta_{m}\right| t_{j}^{m}\right]^{1 / 2}+t_{j}^{m} \rho(\delta),
\end{aligned}
$$

where $\omega, \theta$ are specified by condition $\left(H ; X_{\alpha}\right), \rho$ is defined for the $\theta$ by (1.5), $0 \leqq|\eta|<\delta \leqq T, 0<\varepsilon<\delta-|\eta|$ and $\max \left\{\left|\Delta_{m}\right|\left|\Delta_{n}\right|\right\}<\delta-|\eta|-\varepsilon$. Now let $t, t^{\prime} \in[0, T], \eta=t^{\prime}-t,|\eta|<T, 0<\varepsilon<\varepsilon+\max \left\{\left|\Delta_{m}\right|,\left|\Delta_{n}\right|\right\}<T-|\eta|$, and let $\delta=|\eta|+\varepsilon+\max \left\{\left|\Delta_{m}\right|,\left|\Delta_{n}\right|\right\}+1 / n$. Then $\eta, \varepsilon$, and $\delta$ satisfy the conditions just mentioned above. Hence, letting $m, n \rightarrow \infty, t_{k}^{n} \rightarrow t^{\prime}, t_{j}^{m} \rightarrow t$ in the above estimate, and then, letting $\varepsilon \downarrow 0$ in the resultant inequality, we infer with the aid of (2.1) that

$$
\begin{aligned}
& \exp \left[-2 \omega\left(t+t^{\prime}\right)\right]\left\|u(t)-u\left(t^{\prime}\right)\right\| \\
& \leqq \\
& 2\|x-\tilde{x}\|+\left|t-t^{\prime}\right|(\|\tilde{y}\|+\rho(T))+t \rho\left(\left|t-t^{\prime}\right|+0\right) .
\end{aligned}
$$

Since $\rho(r) \downarrow 0$ as $r \downarrow 0$ and $\tilde{x}$ can be chosen so that $\|\tilde{x}-x\|$ is arbitrarily small, (2.5) implies that $u$ is strongly uniformly continuous on $[0, T)$. Therefore, $u$ is extended uniquely to a function in $\mathcal{C}([0, T] ; X)$ (we denote this by the same symbol $u$ ).

We next show that the extended function $u$ is an integral solution of $(D E)$ constrained in $\left\{\bar{X}_{\gamma, t}\right\}$ for some $\gamma \geqq \alpha$. Let $t \in(0, T]$. In view of (2.1) one finds a sequence $\left\{t_{k(n)}^{n}\right\}$ such that $t_{k(n)}^{n} \uparrow t$ and $u^{n}\left(t_{k(n)}^{n}\right) \rightarrow u(t)$ as $n \rightarrow \infty$ (we take a suitable subsequence $\left\{n_{j}\right\}$ of $\{n\}$ if necessary). Hence by condition $(T)$ there is a number $\gamma \geqq \alpha$ such that $u(t) \in X_{a} \cap \overline{D\left(A_{t}\right)} \subset \bar{X}_{\gamma, t}$, note that $\gamma$ is independent of $t$. This means that $u$ is a weak solution constrained in $\left\{\bar{X}_{\gamma, t}\right\}$. We then demonstrate that $u$ enjoys the integral inequality (2.4). Let $\gamma^{\prime} \geqq \gamma$, $r \in[0, T],(x, y) \in A_{r}, x \in X_{\gamma}$, and let $0 \leqq t<t^{\prime} \leqq T$. Suppose that condition $\left(\boldsymbol{H} ; X_{\gamma^{\prime}}\right)$ is satisfied for some constant $\omega^{\prime} \geqq 0$ and some function $\theta^{\prime} \in \mathcal{I}$. Since $\left(u_{k}^{n}-x\right)-\left(u_{k-1}^{n}-x\right)=h_{k}^{n}\left(v_{k}^{n}+f_{k}^{n}\right)$, we have

$$
\left\|u_{k}^{n}-x\right\|-\left\|u_{k-1}^{n}-x\right\| \leqq h_{k}^{n} \tau_{-}\left(v_{k}^{n}, u_{k}^{n}-x\right)+h_{k}^{n}\left\|f_{k}^{n}\right\|
$$

for $1 \leqq k \leqq N_{n}$. Let $0 \leqq j \leqq i \leqq N_{n}$. Since

$$
\tau_{-}\left(v_{k}^{n}, u_{k}^{n}-x\right)+\tau_{-}\left(-y, u_{k}^{n}-x\right) \leqq \omega^{\prime}\left\|u_{k}^{n}-x\right\|+\theta^{\prime}\left(t_{k}^{n}, r\right)
$$

for $1 \leqq k \leqq N_{n}$, we obtain the estimate

$$
\left\|u_{i}^{n}-x\right\|-\left\|u_{j}^{n}-x\right\| \leqq \sum_{k=j+1}^{i} h_{k}^{n}\left[\tau_{+}\left(y, u_{k}^{n}-x\right)+\omega^{\prime}\left\|u_{k}^{n}-x\right\|+\theta^{\prime}\left(t_{k}^{n}, r\right)+\left\|f_{k}^{n}\right\|\right] .
$$


Letting $t_{i}^{n} \rightarrow t^{\prime}$ and $t_{j}^{n} \rightarrow t$ as $n \rightarrow \infty$, the left side goes to $\left\|u\left(t^{\prime}\right)-x\right\|-\|u(t)-x\|$ and the sum of the terms containing $\left\|f_{k}^{n}\right\|$ 's tends to 0 . On the other hand, the sum of the first three terms on the right side is written as

$$
\int_{t_{j}^{n}}^{t_{i}^{n}}\left[\tau_{+}\left(y, u^{n}(\xi)-x\right)+\omega^{\prime}\left\|u^{n}(\xi)-x\right\|+\theta^{n}(\xi, r)\right] d \xi,
$$

where $u^{n}$ is the step function defined before (2.1) and, likewise, $\theta^{n}$ denotes the step function defined by $\theta^{n}(\xi, r)=\theta^{\prime}\left(t_{k}^{n}, r\right)$ for $\xi \in\left(t_{k-1}^{n}, t_{k}^{n}\right]$ and $1 \leqq k \leqq N_{n}$. Since the integrands of the above integrals $(n \geqq 1)$ are uniformly bounded, the desired integral inequality (2.4) (with $\omega$ and $\theta$ replaced respectively by $\omega^{\prime}$ and $\theta^{\prime}$ ) is obtained by using in the above estimate the upper semicontinuity of the functional $\tau_{+}(\cdot, \cdot)$, the upper semicontinuity of $\theta^{\prime}(\cdot, r)$ and then applying the Lebesgue dominated convergence theorem.

Finally, we mimic the proof of Benilan's uniqueness theorem to show that for every $\gamma^{\prime} \geqq \gamma$ the function $u$ is the only element of $\mathcal{G}_{\gamma^{\prime}}[0, T]$ satisfying $u(0)=x$. Let $\tilde{u}$ be any element of $\mathcal{G}_{\gamma^{\prime}}[0, T]$ and let $0 \leqq s \leqq s^{\prime} \leqq T$. Then, substituting $\tilde{u}, s^{\prime}, s$, and $\left(u_{k}^{n}, v_{k}^{n}\right)$ respectively into $u, t^{\prime}, t$, and $(x, y)$ on the inequality (2.4), one has

$$
\begin{aligned}
& \left\|\tilde{u}\left(s^{\prime}\right)-u_{k}^{n}\right\|-\left\|\tilde{u}(s)-u_{k}^{n}\right\| \\
\leqq & \int_{s}^{s^{\prime}}\left[\tau_{+}\left(v_{k}^{n}, \tilde{u}(\xi)-u_{k}^{n}\right)+\tilde{\omega}\left\|\tilde{u}(\xi)-u_{k}^{n}\right\|+\theta\left(\xi, t_{k}^{n}\right)\right] d \xi,
\end{aligned}
$$

where both $\tilde{\boldsymbol{\omega}} \geqq 0$ and $\tilde{\theta} \in \mathcal{I}$ depend only on $\tilde{u}$. Since $h_{k}^{n} \tau_{+}\left(v_{k}^{n}, \tilde{u}(\xi)-u_{k}^{n}\right) \leqq$ $\left\|\tilde{u}(\xi)-u_{k-1}^{n}\right\|-\left\|\tilde{u}(\xi)-u_{k}^{n}\right\|+h_{k}^{n}\left\|f_{k}^{n}\right\|$, we see in the same way as in the preceding paragraph that the inequality

$$
\begin{aligned}
& \int_{t_{j}^{n}}^{t_{i}^{n}}\left(\left\|\tilde{u}\left(s^{\prime}\right)-u^{n}(\eta)\right\|-\left\|\tilde{u}(s)-u^{n}(\eta)\right\|\right) d \eta \\
\leqq & \int_{s}^{s^{\prime}}\left(\left\|\tilde{u}(\xi)-u^{n}\left(t_{j}^{n}\right)\right\|-\left\|\tilde{u}(\xi)-u^{n}\left(t_{i}^{n}\right)\right\|\right) d \xi \\
+ & \int_{t_{j}^{n}}^{t_{i}^{n}} \int_{s}^{s^{\prime}}\left[\tilde{\omega}\left\|\tilde{u}(\xi)-u^{n}(\eta)\right\|+\widetilde{\theta}^{n}(\xi, \eta)\right] d \xi d \eta+\left(s^{\prime}-s\right) \sum_{k=j+1}^{i} h_{k}^{n}\left\|f_{k}^{n}\right\|
\end{aligned}
$$

holds for $0 \leqq j \leqq i \leqq N_{n}$ and $n \geqq 1$, where $\widetilde{\theta}^{n}(n \geqq 1)$ are functions defined by $\tilde{\theta}^{n}(\xi, \eta)=\widetilde{\theta}\left(\xi, t_{k}^{n}\right)$ for $\eta \in\left(t_{k-1}^{n}, t_{k}^{n}\right]$ and $1 \leqq k \leqq N_{n}$. Letting $t_{i}^{n} \rightarrow t^{\prime}$ and $t_{j}^{n} \rightarrow t$ as $n \rightarrow \infty$, we have

$$
\begin{aligned}
& \int_{t}^{t^{\prime}}\left(\left\|\tilde{u}\left(s^{\prime}\right)-u(\eta)\right\|-\|\tilde{u}(s)-u(\eta)\|\right) d \eta \\
+ & \int_{s}^{s^{\prime}}\left(\left\|\tilde{u}(\xi)-u\left(t^{\prime}\right)\right\|-\|\tilde{u}(\xi)-u(t)\|\right) d \xi \\
\leqq & \tilde{\omega} \int_{t}^{t^{\prime}} \int_{s}^{s^{\prime}}\|\tilde{u}(\xi)-u(\eta)\| d \xi d \eta+\int_{t}^{t^{\prime}} \int_{s}^{s^{\prime}} \tilde{\theta}(\xi, \eta) d \xi d \eta .
\end{aligned}
$$


We now apply proposition B of [17] (Proposition 2.5 below). Putting $[a, b]=[0, T], \phi\left(t^{\prime}, t\right)=\left\|\tilde{u}\left(t^{\prime}\right)-u(t)\right\|, c=\tilde{\omega}, d=1$ and $\psi\left(t^{\prime}, t\right)=\tilde{\theta}\left(t^{\prime}, t\right)$ in Proposition 2.5 below, we have

$$
e^{-\tilde{\omega}_{t^{\prime}}}\left\|\tilde{u}\left(t^{\prime}\right)-u\left(t^{\prime}\right)\right\| \leqq e^{-\tilde{\omega} t}\|\tilde{u}(t)-u(t)\| \quad \text { for } \quad 0 \leqq t \leqq t^{\prime} \leqq T,
$$

which means that $u$ is the only integral solution constrained in $\left\{\bar{X}_{\boldsymbol{\gamma}^{\prime}, t}\right\}$ and satisfying $u(0)=x$.

q.e.d.

REMARK 2.1. In the above theorem we assumed that the convergence (2.1) held. However, as will be seen in Section 3, (2.1) is always valid for every consistent discrete scheme $((D S))$ for $(D E)$. Accordingly Theorem 2.4 states that the limit function $u$ of $(2.1)$ is uniquely determined in the class $\mathcal{G}_{y}[0, T]$ $(\gamma \geqq \alpha)$ and does not depend upon the choice of discrete scheme $((D S))$ as far as the consistency condition (C) holds for the scheme.

Finally, we mention the full statement of Proposition B quoted in the proof of Theorem 2.4 since it is also used in the proof of Theorem 2.7 below.

Proposition 2.5. Let $a<b$ and let $\phi, \psi$ be nonnegative functions defined on all of $[a, b] \times[a, b]$ satisfying the following conditions:

$\left(1^{0}\right) \phi$ is continuous over $[a, b] \times[a, b]$.

$\left(2^{\circ}\right) \quad \psi$ is upper semicontinuous on $[a, b] \times[a, b]$.

(3) there exist constants $c, d \geqq 0$ such that for $a \leqq s<t \leqq b$ and $a \leqq \sigma<\tau \leqq b$ we have

$$
\begin{aligned}
& \int_{\sigma}^{\tau}[\phi(\xi, t)-\phi(\xi, s)] d \xi+\int_{s}^{t}[\phi(\tau, \eta)-\phi(\sigma, \eta)] d \eta \\
\leqq & \int_{s}^{t} \int_{\sigma}^{\tau} \phi(\xi, \eta) d \xi d \eta+d \int_{s}^{t} \int_{\sigma}^{\tau} \psi(\xi, \eta) d \xi d \eta .
\end{aligned}
$$

Then we have

$$
e^{-c t} \phi(t, t)-e^{-c s} \phi(s, s) \leqq d e^{-c s} \int_{s}^{t} \psi(\xi, \xi) d \xi \quad \text { for } \quad s \leqq t \leqq b .
$$

If in particular $\Psi(s, s)=0$ for $s \in[a, b]$, then

$$
e^{-c t} \phi(t, t) \leqq e^{-c s} \phi(s, s) \quad \text { for } \quad a \leqq s \leqq t \leqq b .
$$

2.3. Lipschitz continuity of weak solutions.

In the preceding section we have shown that a weak solution with a given initial-value of $(D E)$ constrained in some $X_{\infty}$ is unique in any class $\mathcal{G}_{\gamma}[0, T]$ $(\gamma \geqq \alpha)$. Here some other aspects of the integral solution are given and the Lipschitz continuity of weak solutions is discussed through Proposition 2.5.

In what follows, we assume that $\tau_{+}$and $\tau_{-}$denote the tangent functionals of the unit ball of $X^{* *}$ and the restriction of $A_{t}$ to $X_{\infty}$ is denoted by $A_{t, \infty}$. For $\alpha>0$ and $t \in[0, T]$ we introduce two operators $\hat{A}_{t, \infty}$ and $\mathcal{A}_{t, \alpha}$. 
Definition 2.3. Let $\alpha>0$. We say that $(u, v) \in \hat{A}_{t, \infty}$ if $(u, v) \in \bar{X}_{\alpha, t} \times \bar{X}^{* *}$ and

$$
\tau_{-}(v, u-x)+\tau_{-}(-y, u-x) \leqq \omega_{\infty}\|u-x\|+\theta_{\infty}(t, s)
$$

for $s \in[0, T]$ and $(x, y) \in A_{s, a}$, where $\omega_{\infty} \geqq 0$ and $\theta_{\infty} \in \mathcal{I}$ are furnished by condition $\left(\boldsymbol{H} ; X_{\boldsymbol{s}}\right)$. We say that $(z, w) \in \mathcal{A}_{t, \infty}$ if $(z, w) \in \hat{A}_{t, \infty}$ and for $s \in[0, T]$ and $(u, v) \in \hat{A}_{s, a}$, we have

$$
\tau_{-}(w, z-u)+\tau_{-}(-v, z-u) \leqq \omega_{a}\|z-u\|+\theta_{a}(t, s) .
$$

It is easily seen that $\bar{A}_{t, \infty} \subset \mathcal{A}_{t, \infty} \subset \hat{A}_{t, \infty}$ for $t \in[0, T]$ and the family $\left\{\mathscr{A}_{t, \infty}\right.$ : $t \in[0, T]\}$ also satisfies condition $\left(H ; X_{\infty}\right)$ for the same $\omega_{\infty}$ and $\theta_{a}$ as in $\left(H ; X_{\infty}\right)$ that is imposed for the original family $\left\{A_{t, \infty}\right\}$. Using the above operators, one can introduce another notion of integral solution in a slightly stronger sense: Let $\alpha>0$. An $X$-valued function $u$ in $\mathcal{C}([0, T] ; X)$ is said to belong to the class $\hat{\mathcal{G}}_{\omega}$, if $u \in \mathcal{I}_{\omega}$ and there exists an $\omega \geqq 0$ and $\theta \in \mathcal{I}$ such that for $r \in[0, T]$, $(x, y) \in \hat{A}_{r, \infty}$ and $0 \leqq t \leqq t^{\prime} \leqq T$, one has

$$
\begin{aligned}
& \left\|u\left(t^{\prime}\right)-x\right\|^{2}-\|u(t)-x\|^{2} \\
\leqq & 2 \int_{t}^{t^{\prime}}\left[\tau_{+}(y, u(\xi)-x)+\omega\|u(\xi)-x\|+\theta(\xi, r)\right] d \xi .
\end{aligned}
$$

As is seen from the proof of Theorem 2.4, a weak solution $u$ obtained by (2.1) satisfies $(2.4)^{*}$ with $\alpha$ replaced by some $\gamma$ and belongs to the class $\hat{\mathcal{G}}_{\gamma}$. Since $\tau_{+}$agrees on $X \times X$ with the tangent functional of the unit ball of $X$, condition (2.4)* is stronger than (2.4). We then give a result analogous to Bénilan [1], Théorème 1.2 on Page 1.7. To this end, we use the space $\boldsymbol{A} \boldsymbol{C} \equiv \boldsymbol{A} \boldsymbol{C}([0, T] ; X)$ of all $X$-valued, strongly absolutely continuous functions on $[0, T]$ and, for every $u \in \boldsymbol{A C}$, we introduce a (possibly multi-valued) function $D^{+} u$ from $[0, T]$ into $X^{* *}$ in the following way: Given $u \in A C$ there is a Lebesgue null set $N$ in $[0, T]$ such that

$$
\lim _{h \rightarrow \infty} \sup |h|^{-1}|| u(t+h)-u(t) \|<\infty \quad \text { for } \quad t \in[0, T]-N .
$$

Hence for each $t \in(0, T)-N$, the set

$$
D^{+} u(t)=\bigcap_{\varepsilon>0} \overline{c o}^{\sigma\left(X^{* *}, X^{*}\right)}\left\{h^{-1}[u(t+h)-u(t)]: 0<h<\varepsilon\right\},
$$

is nonempty, where $\overline{c o}^{\sigma\left(X^{* *}, X^{*}\right)}[W]$ means the weak* convex closure in $X^{* *}$ of a set $W$. The set $D^{+} u(t)$ may be regarded as a weak* right-derivative of $u$ at $t$ in a generalized sense.

Proposition 2.6. Let $u \in \hat{\mathcal{G}}_{\text {s. }}$. If $u \in A C([0, T] ; X)$, then it satisfies the relation $D^{+} u(t) \subset \mathcal{A}_{t, \infty} u(t)$ (a generalized form of equation $(D E)$ ) for a.e. $t \in[0, T]$. 
Proof. Let $u \in \hat{\mathcal{I}}_{\infty}$ and assume that $u \in \boldsymbol{A C}$. Since $\hat{\mathcal{G}}_{\infty} \subset \mathcal{I}_{\infty}, u(t)$ lies in $\bar{X}_{\omega, t}$ for $t \in[0, T]$ and there is a constant $\omega \geqq 0$ and a function $\theta \in \mathcal{I}$ for which we have (in the same way as in the proof of Proposition 2.3)

$$
\begin{aligned}
& \tau_{+}\left(h^{-1}[u(t+h)-u(t)], u(t)-x\right) \leqq h^{-1}[\|u(t+h)-x\|-\|u(t)-x\|] \\
\leqq & h^{-1} \int_{t}^{t+h}\left[\tau_{+}(y, u(\xi)-x)+\omega\|u(\xi)-x\|+\theta(\xi, r)\right] d \xi
\end{aligned}
$$

for $r \in[0, T], t \in[0, T),(x, y) \in A_{r, \omega}$ and $h>0$ with $t+h \leqq T$. Now let $N$ be the null set as mentioned before (2.9), $t \in[0, T)-N$, and let $v(t) \in D^{+} u(t)$. Then the application of the upper semicontinuity of $\tau_{+}$as well as that of $\theta(\cdot, r)$ to the estimate $(2.10)$ yields

$$
\tau_{-}(v(t), u(t)-x)+\tau_{-}(-y, u(t)-x) \leqq \omega\|u(t)-x\|+\theta(t, r)
$$

for $(x, y) \in A_{r, \infty}$. This means that $(u(t), v(t)) \in A_{t, \infty}$. Next, in view of $(2.4)^{*}$, we see that $(2.10)$ holds with $(x, y) \in \hat{A}_{r, \infty}$ replaced by $(x, y) \in \hat{A}_{r, \infty}$. So, we get

$$
\tau_{-}(v(t), u(t)-x)+\tau_{-}(-y, u(t)-x) \leqq \omega\|u(t)-x\|+\theta(t, r)
$$

for $r \in[0, T]$ and $(x, y) \in \hat{A}_{r, a}$. From this it follows that $(u(t), v(t)) \in \mathscr{A}_{t, \infty}$. Thus $D^{+} u(t) \subset \mathcal{A}_{t, \infty} u(t)$.

q.e.d.

We now discuss the Lipschitz continuity of weak solutions.

Theorem 2.7. Let $u$ be a weak solution of $(D E)$ with $(I C)$ as mentioned in Theorem 2.4, $\gamma$ a number for which $u$ belongs to $\mathcal{I}_{\gamma}[0, T]$, and let $\omega \geqq 0, \theta \in \mathbb{I}$ be furnished by condition $\left(\boldsymbol{H} ; X_{\gamma}\right)$. If $\theta \in I_{B V}$ and $\|u(h)-x\|=0(h)$ as $h \downarrow 0$, then $u$ is Lipschitz continuous on $[0, T]$.

Proof. Let $h \in(0, T / 2)$ and set $t_{k}=k h$ for $0 \leqq k \leqq N=[(T-h) / h]$. By Theorem 2.4, the restriction of $u$ on $[h, T]$ is an integral solution of $(D E)_{h}$ constrained in $\left\{\bar{X}_{\gamma, t}: t \in[h, T]\right\}$. Set $\tilde{u}(\xi)=u(\xi+h)$ for $\xi \in[0, T-h]$. Then $\tilde{u}$ satisfies

$$
\begin{aligned}
& \left\|\tilde{u}\left(t^{\prime}\right)-x\right\|-\|\tilde{u}(t)-x\| \\
\leqq & \int_{t}^{t^{\prime}}[\tau(\tilde{u}(\xi)-x, y)+\omega\|\tilde{u}(\xi)-x\|+\theta(\xi+h, r)] d \xi
\end{aligned}
$$

for $0 \leqq t<t^{\prime} \leqq T-h,(x, y) \in A_{r}, x \in X_{\gamma}$ and $r \in[0, T]$. Hence we see in the same way as in the derivation of (2.7) that the integral inequality (2.8) holds with $[a, b]=[0, T-h], \phi(\xi, \eta)=\|\tilde{u}(\xi)-u(\eta)\|, \psi(\xi, \eta)=\theta(\xi+h, \eta), c=\omega$ and with $d=1$ for $0 \leqq t \leqq t^{\prime} \leqq T-h$ and $0 \leqq \tau \leqq \tau^{\prime} \leqq T-h$. Therefore the application of Proposition 2.5 yields that for every $k$ with $1 \leqq k \leqq N$ we have

$$
\begin{aligned}
& \exp \left(-\omega t_{k}\right)\left\|u\left(t_{k+1}\right)-u\left(t_{k}\right)\right\|-\exp \left(-\omega t_{k-1}\right)\left\|u\left(t_{k}\right)-u\left(t_{k-1}\right)\right\| \\
\leqq & \exp \left(-\omega t_{k-1}\right) \int_{0}^{h} \theta\left(t_{k}+\zeta ; t_{k-1}+\zeta\right) d \zeta .
\end{aligned}
$$


Now let $\boldsymbol{M}(\geqq 0)$ be a constant associated through (1.6) with $\theta \in \mathcal{I}_{\boldsymbol{B V}}$. Then we have $\sum_{j=1}^{k} \theta\left(t_{j}+\zeta ; t_{j-1}+\zeta\right) \leqq M$ for a.e. $\zeta \in[0, h]$ and so $\sum_{j=1}^{k} \int_{0}^{h} \theta\left(t_{j}+\zeta\right.$; $\left.t_{j-1}+\zeta\right) d \zeta \leqq M h$. Suppose that $\|u(h)-x\| \leqq L h$ for $h$ sufficiently small and some constant $\boldsymbol{L}(>0)$. Then (2.11) implies

$$
\begin{aligned}
& \left\|u\left(t_{k+1}\right)-u\left(t_{k}\right)\right\| \\
\leqq & e^{\omega h k} \|\left[u(h)-x \|+\sum_{j=1}^{k} \int_{0}^{h} \theta\left(t_{j}+\zeta ; t_{j-1}+\zeta\right) d \zeta\right] \leqq e^{\omega h k}(\boldsymbol{L}+\boldsymbol{M}) h
\end{aligned}
$$

for $1 \leqq k \leqq N$. Since $h>0$ is arbitrary and $u \in C([0, T] ; X)$, the above estimate yields that $u$ is Lipschitz continuous over $[0, T]$ with Lipschitz constant $e^{\omega T}(\boldsymbol{L}+\boldsymbol{M})$.

q.e.d.

We shall show in the next section (Remark 3.3) that $\|u(h)-x\|=0(h)$ as $h \downarrow 0$ if $x \in D_{a}\left(\mathcal{A}_{0, a}\right)$ in the above theorem. It turns out that we can obtain a natural sufficient condition for a weak solution to be Lipschitz continuous on $[0, T]$.

\section{Discrete approximation}

In this section we assume that there exists an l.s.c. functional $\mathfrak{p}: X \rightarrow[0, \infty]$ satisfying $(\boldsymbol{P})$ and $(\boldsymbol{T})$ and, under the hypothesis $(\boldsymbol{H})$, we give basic estimates for the consistent discrete schemes for $(D E)_{s}$ as mentioned in Section 2.1 and establish the convergence (2.1).

In what follows, we restrict ourselves to a fixed set $X_{\infty}$ and assume that the family of operators $\left\{A_{t}\right\}$ satisfies condition $\left(\boldsymbol{H} ; X_{\infty}\right)$. Hence, for brevity in notation, the symbols $\omega, \theta$ and $\rho$ are used to denote respectively the constant $\omega_{\infty} \geqq 0$ and the functions $\theta_{a}$ and $\rho_{a}$ that are furnished by condition $\left(\boldsymbol{H} ; X_{a}\right)$. Likewise, the restriction $A_{t, a}$ of the operator $A_{t}$ to $X_{a}$ is denoted simply by the same symbol $A_{t}$. Let $\beta \in(0, \alpha], s, \hat{s} \in[0, T), x \in \bar{X}_{\beta, s}, \hat{x} \in \bar{X}_{\beta, \hat{s} s}$, and suppose that there exist two discrete schemes $((D S))_{s} \equiv\left(\left\{\Delta_{n}\right\},\left\{\left(u_{k}^{n}, v_{k}^{n}\right)\right\},\left\{f_{k}^{n}\right\}\right)$ and $((D S))_{\hat{s}} \equiv\left(\left\{\hat{\Delta}_{m}\right\},\left\{\left(\hat{u}_{j}^{m}, \hat{v}_{j}^{m}\right)\right\},\left\{\hat{f}_{j}^{m}\right\}\right)$ in $X_{\infty}$ which are consistent respectively with $(D E)_{s}$ and $(D E)_{\hat{s}}$. Namely,

(i) $\Delta_{n}=\left\{s=t_{0}^{n}<t_{1}^{n}<\cdots<t_{N_{n}}^{n} \leqq t_{N_{n}+1}^{n}=T\right\} \quad(n \geqq 1)$, $\hat{\Delta}_{m}=\left\{\hat{s}=\hat{t}_{0}^{m}<\hat{t}_{1}^{m}<\cdots<\hat{t}_{\hat{N}_{m}} \leqq \hat{t}_{\hat{N}_{m}+1}^{m}=T\right\} \quad(m \geqq 1) ;$

(ii) the sequences $\left\{u_{k}^{n}\right\},\left\{v_{k}^{n}\right\},\left\{f_{k}^{n}\right\},\left\{\hat{u}_{j}^{m}\right\},\left\{\hat{v}_{j}^{m}\right\}$ and $\left\{\hat{f}_{j}^{m}\right\}$ in $X$ satisfy the difference equations

$$
\begin{array}{lll}
((D E))_{n} & \left(h_{k}^{n}\right)^{-1}\left[u_{k}^{n}-u_{k-1}^{n}\right]=v_{k}^{n}+f_{k}^{n}, h_{k}^{n}=t_{k}^{n}-t_{k-1}^{n},\left(u_{k}^{n}, v_{k}^{n}\right) \in A_{t_{k}^{n}} & \left(1 \leqq k \leqq N_{n}\right) . \\
((D E))_{m} & \left(\hat{h}_{j}^{m}\right)^{-1}\left[\hat{u}_{j}^{m}-\hat{u}_{j-1}^{m}\right]=\hat{v}_{j}^{m}+\hat{f}_{j}^{m}, \hat{h}_{j}^{m}=\hat{t}_{j}^{m}-\hat{t}_{j-1}^{m},\left(\hat{u}_{j}^{m}, \hat{v}_{j}^{m}\right) \in A_{\hat{t}_{j}^{m}} & \left(1 \leqq j \leqq \hat{N}_{m}\right)
\end{array}
$$

for $m, n \geqq 1$ as well as the consistency conditions 


$$
\begin{array}{ll}
\lim _{n \rightarrow \infty} u_{0}^{n}=x, & \lim _{n \rightarrow \infty}\left|\Delta_{n}\right|=0, \quad \lim _{n \rightarrow \infty} \sum_{k=1}^{N_{n}} h_{k}^{n}|| f_{k}^{n} \|=0, \\
\lim _{m \rightarrow \infty} \hat{u}_{0}^{m}=x, & \lim _{m \rightarrow \infty}\left|\hat{\Delta}_{m}\right|=0, \quad \lim _{m \rightarrow \infty} \sum_{j=1}^{N_{m}} \hat{h}_{j}^{m}|| \hat{f}_{j}^{m} \|=0 ; \quad \text { and }
\end{array}
$$

(iii) both $\left\{\left(u_{k}^{n}, v_{k}^{n}\right)\right\}$ and $\left\{\left(\hat{u}_{j}^{m}, \hat{v}_{j}^{m}\right)\right\}$ are sequences of elements in $X_{\infty} \times X$.

We now wish to estimate the difference between $u_{k}^{n}$ and $\hat{u}_{j}^{m}$. Firstly, the following facts are obtained in the same way as in Kobayashi [16], Lemma 1.1.

Lemma 3.1. Let $t, \tilde{t} \in[0, T],(u, v) \in A_{t}$ and $(\tilde{x}, \tilde{y}) \in A_{\tilde{t}} . \quad$ The following are equivalent :

(3.1) $\quad \tau_{-}(v, u-\tilde{x})+\tau_{-}(-\tilde{y}, u-\tilde{x}) \leqq \omega\|u-\tilde{x}\|+\theta(t, \tilde{t})$;

(3.2) $(\lambda+\mu-\lambda \mu \omega)\|u-\tilde{x}\| \leqq \lambda\|u-\tilde{x}-\mu v\|+\mu\|\tilde{x}-u-\lambda \tilde{y}\|+\lambda \mu \theta(t, \tilde{t})$,

$$
(2-\lambda \omega)\|u-\tilde{x}\| \leqq\|u-\tilde{x}-\lambda v\|+\|\tilde{x}-u-\lambda \tilde{y}\|+\lambda \theta(t, \tilde{t}), \quad(\lambda>0) .
$$

Moreover, (3.3) implies that

$$
(1-\lambda \omega)\|\tilde{x}-u\| \leqq\|u-\tilde{x}-\lambda v\|+\lambda\|\tilde{y}\|+\lambda \theta(t, \tilde{t}), \quad(\lambda>0)
$$

Secondly, we prepare two technical lemmas which will be used later.

Lemma 3.2. Let $\tilde{t} \in[0, T]$ and $(\tilde{x}, \tilde{y}) \in A_{\tilde{t}}$. Let $\lambda>0, w \in X$, and set $z=\tilde{x}-\lambda(\tilde{y}+w)$. Then, for $1 \leqq k \leqq N_{n}$, we have

$$
\begin{gathered}
\left(\lambda+h_{k}^{n}-\lambda h_{k}^{n} \omega\right)\left\|u_{k}^{n}-\tilde{x}\right\| \leqq \lambda\left\|u_{k-1}^{n}-\tilde{x}\right\|+h_{k}^{n}\left\|u_{k}^{n}-z\right\| \\
+\lambda h_{k}^{n}\left[\left\|f_{k}^{n}\right\|+\|w\|+\theta\left(t_{k}^{n}, \tilde{t}\right)\right] .
\end{gathered}
$$

Proof. Let $1 \leqq k \leqq N_{n}$. Since $t^{-1}(\|u\|-\|u-t v\|) \leqq \tau_{-}(u, v)$ for $t>0$, we get

$\left(h_{k}^{n}\right)^{-1}\left(\left\|u_{k}^{n}-\tilde{x}\right\|-\left\|u_{k-1}^{n}-\tilde{x}\right\|\right)+\lambda^{-1}\left(\left\|u_{k}^{n}-\tilde{x}\right\|-\left\|u_{k}^{n}-z\right\|\right)-\left\|f_{k}^{n}\right\|$

$$
\leqq\left(h_{k}^{n}\right)^{-1}\left(\left\|u_{k}^{n}-\tilde{x}\right\|-\left\|u_{k-1}^{n}+h_{k}^{n} f_{k}^{n}-\tilde{x}\right\|\right)+\lambda^{-1}\left(\left\|u_{k}^{n}-\tilde{x}\right\|-\left\|u_{k}^{n}-\tilde{x}-\lambda(-\tilde{y}-w)\right\|\right)
$$$$
\leqq\left(h_{k}^{n}\right)^{-1}\left(\left\|u_{k}^{n}-x\right\|-\left\|u_{k}^{n}-\tilde{x}-h_{k}^{n} v_{k}^{n}\right\|\right)+\lambda^{-1}\left(\left\|u_{k}^{n}-\tilde{x}\right\|-\left\|u_{k}^{n}-\tilde{x}-\lambda(-\tilde{y})\right\|\right)+\|w\| .
$$$$
\leqq \tau_{-}\left(v_{k}^{n}, u_{k}^{n}-\tilde{x}\right)+\tau_{-}\left(-\tilde{y}, u_{k}^{n}-\tilde{x}\right)+\|w\| \leqq \omega\left\|u_{k}^{n}-x\right\|+\theta\left(t_{k}^{n}, \tilde{t}\right)+\|w\| \text {. }
$$

From this we have the assertion.

q.e.d.

Lemma 3.3. Let $\tilde{t} \in[0, T]$ and $(\tilde{x}, \tilde{y}) \in A_{\tilde{t}}$. Then we have

$$
\prod_{p=1}^{k}\left(1-h_{p}^{n} \omega\right)\left\|u_{k}^{n}-\tilde{x}\right\| \leqq\left\|u_{0}^{n}-\tilde{x}\right\|+\left(t_{k}^{n}-s\right)\|\tilde{y}\|+\sum_{p=1}^{k} h_{p}^{n}\left[\left\|f_{p}^{n}\right\|+\theta\left(t_{p}^{n}, \tilde{t}\right)\right]
$$

for $1 \leqq k \leqq N_{n}$ and $n$ with $\left|\Delta_{n}\right|<1 / \omega$.

Proof. Since $\left\{A_{t}\right\}$ satisfies $\left(H ; X_{\omega}\right),(3.4)$ of Lemma 3.1 yields 


$$
\begin{aligned}
& \left(1-h_{k}^{n} \omega\right)\left\|u_{k}^{n}-\tilde{x}\right\| \leqq \\
= & \| u_{k}^{n}-\tilde{x}-\left(u_{k}^{n}-\tilde{x}-u_{k-1}^{n}-h_{k}^{n} f_{k}^{n}\|\|+h_{k}^{n}\|\tilde{y}\|+h_{k}^{n}\|\tilde{y}\|+h_{k}^{n} \theta\left(t_{k}^{n}, \tilde{t}\right)\right. \\
\leqq & \left\|u_{k-1}^{n}-\tilde{x}\right\|+h_{k}^{n}\left[\left\|f_{k}^{n}\right\|+\|\tilde{t}\|+\theta\left(t_{k}^{n}, \tilde{t}\right)\right] \quad \text { for } \quad 1 \leqq k \leqq N_{n} .
\end{aligned}
$$

In view of this, we obtain the desired estimate by induction.

q.e.d.

Thirdly, let $1 \leqq k \leqq N_{n}$ and $1 \leqq j \leqq \hat{N}_{m}$. Setting $\tilde{x}=\hat{u}_{j}^{m}, \tilde{y}=\hat{v}_{j}^{m}, \lambda=\hat{h}_{j}^{m}$ and $w=\hat{f}_{j}^{m}$ in Lemma 3.2 , we have the following estimate which plays a central role in the argument below:

$$
\begin{aligned}
& \left(h_{k}^{n}+\hat{h}_{j}^{m}-h_{k}^{n} \hat{h}_{j}^{m} \omega\right)\left\|u_{k}^{n}-\hat{u}_{j}^{m}\right\| \\
\leqq & \hat{h}_{j}^{m}\left\|u_{k-1}^{n}-\hat{u}_{j}^{m}\right\|+h_{k}^{n}\left\|u_{k}^{n}-\hat{u}_{j-1}^{m}\right\|+h_{k}^{n} \hat{h}_{j}^{m}\left[\left\|f_{k}^{n}\right\|+\left\|\hat{f}_{j}^{m}\right\|+\theta\left(t_{k}^{n}, \hat{t}_{j}^{m}\right)\right]
\end{aligned}
$$

Here it is convenient to employ the following notation:

$$
\begin{aligned}
& a_{k, j}=\left\|u_{k}^{n}-\hat{u}_{j}^{m}\right\|, \quad \nu_{k}=\left\|f_{k}^{n}\right\|, \quad \hat{\nu}_{j}=\left\|\hat{f}_{j}^{m}\right\|, \\
& d_{k, j}=\theta\left(t_{k}^{n}, \hat{t}_{j}^{m}\right)\left(\leqq \rho\left(\left|t_{k}^{n}-\hat{t}_{j}^{m}\right|\right)\right), \\
& \alpha_{k, j}=\hat{h}_{j}^{m} /\left(h_{k}^{n}+\hat{h}_{j}^{m}\right), \quad \beta_{k, j}=h_{k}^{n} /\left(h_{k}^{n}+\hat{h}_{j}^{m}\right), \quad \gamma_{k, j}=h_{k}^{n} \hat{h}_{j}^{m} /\left(h_{k}^{n}+\hat{h}_{j}^{m}\right),
\end{aligned}
$$

where the suffices $m$ and $n$ are eliminated since it will cause no confusion. Hence (3.5) can be rewritten as

$$
a_{k, j} \leqq \alpha_{k, j} a_{k-1, j}+\beta_{k, j} a_{k, j-1}+\gamma_{k, j}\left(\omega a_{k, j}+\nu_{k}+\hat{\nu}_{j}+d_{k, j}\right) .
$$

Fourthly, for $1 \leqq k \leqq N_{n}, 1 \leqq j \leqq \hat{N}_{m}$ and $\eta \in R$, set

$$
c_{k, j}(\eta)=\left[\left(t_{k}^{n}-\hat{t}_{j}^{m}-\eta\right)^{2}+\left|\Delta_{n}\right|\left(t_{k}^{n}-s\right)+\left|\hat{\Delta}_{m}\right|\left(\hat{t}_{j}^{m}-\hat{s}\right)\right]^{1 / 2} .
$$

Then we see in a way similar to Kobayashi [16], p. 649, that the inequality

$$
\alpha_{k, j} c_{k-1, j}(\eta)+\beta_{k, j} c_{k, j-1}(\eta) \leqq c_{k, j}(\eta)
$$

holds for $1 \leqq k \leqq N_{n}$ and $1 \leqq j \leqq \hat{N}_{m}$.

Finally, noting that $\rho$ is monotone nondecreasing over $[0, T]$, we infer that if $0<\kappa<\delta \leqq T$ and $r^{\prime} \in[0, \delta-\kappa)$, the function $\rho$ satisfies the inequality

$$
\rho(r) \leqq \kappa^{-1} \rho(T)\left|r-r^{\prime}\right|+\rho(\delta) \quad \text { for } \quad r \in[0, T] .
$$

Using the estimates (3.6) through (3.8), we obtain the following result.

Lemma 3.4. Let $0 \leqq|\eta|<\delta \leqq T, 0<\kappa<\delta-|\eta|$, and let, $m, n$ be such that $\left|\hat{\Delta}_{m}\right|,\left|\Delta_{n}\right|<\min \{\delta-|\eta|-\kappa, 1 / 2 \omega\}$. Then for every $r \in[0, T]$ and every $(\tilde{x}, \tilde{y}) \in A_{r}$, we have 


$$
\begin{aligned}
& \prod_{p=1}^{k}\left(1-\omega h_{q}^{n}\right) \prod_{q=1}^{j}\left(1-\omega \hat{h}_{q}^{m}\right) a_{k, j} \\
\leqq & \left\|u_{0}^{n}-\tilde{x}\right\|+\left\|\hat{u}_{0}^{m}-\tilde{x}\right\|+c_{k, j}(s-\hat{s})[\|\tilde{y}\|+\rho(T)] \\
+ & \sum_{p=1}^{k} h_{p}^{n} \nu_{p}+\sum_{q=1}^{j} \hat{h}_{q}^{m} \hat{\nu}_{q}+\left(\hat{t}_{j}^{m}-\hat{s}\right)\left[\kappa^{-1} \rho(T) c_{k, j}(\eta)+\rho(\delta)\right]
\end{aligned}
$$

for $0 \leqq k \leqq N_{n}$ and $0 \leqq j \leqq \hat{N}_{m}$, where $\prod_{p=1}^{0}\left(1-\omega h_{p}^{n}\right)=1$ and $\prod_{q=1}^{0}\left(1-\omega \hat{h}_{q}^{m}\right)=1$.

Proof. First the application of Lemma 3.3 with $\tilde{t}=r$ yields

$$
\prod_{p=1}^{k}\left(1-h_{p}^{n} \omega\right) a_{k, 0} \leqq\left\|u_{0}^{n}-\tilde{x}\right\|+\left\|\tilde{x}-\hat{u}_{0}^{m}\right\|+c_{k, 0}(s-\hat{s})[\|\tilde{y}\|+\rho(T)]+\sum_{p=1}^{k} h_{p}^{n} \nu_{p},
$$

so (3.9) holds for $0 \leqq k \leqq N_{n}$ and $j=0$. Next, Lemma 3.3 is still valid even if $u_{k}^{n}, t_{k}^{n}, h_{k}^{n}$ and $\varepsilon_{k}^{n}$ are replaced by $\hat{u}_{j}^{m}, \hat{t}_{j}^{m}, \hat{h}_{j}^{m}$ and $\hat{\varepsilon}_{j}^{m}$, respectively. This means that (3.9) holds for $k=0$ and $0 \leqq j \leqq \hat{N}_{m}$. Now let $1 \leqq k \leqq N_{n}, 1 \leqq j \leqq \hat{N}_{m}$, and suppose that (3.9) holds for the pairs $(k-1, j)$ and $(k, j-1)$. We want to show that (3.9) holds for the pair $(k, j)$ under this assumption. Once this is done, then it is concluded by induction that (3.9) holds for all pairs $(k, j)$ with $0 \leqq k \leqq N_{n}$ and $0 \leqq j \leqq \hat{N}_{m}$. Using (3.6), one has

$$
\begin{gathered}
\omega_{k, j}\left(1-\gamma_{k, j} \omega\right) a_{k, j} \leqq\left(1-h_{k}^{n} \omega\right) \alpha_{k, j} \omega_{k-1, j} a_{k-1, j} \\
+\left(1-\hat{h}_{j}^{m} \omega\right) \beta_{k, j} \omega_{k, j-1} a_{k, j-1}+\omega_{k, j} \gamma_{k, j}\left(\nu_{k}+\hat{\nu}_{j}+d_{k, j}\right),
\end{gathered}
$$

where $\omega_{k, j}=\prod_{p=1}^{k}\left(1-h_{p}^{n} \omega\right) \cdot \prod_{q=1}^{j}\left(1-\hat{h}_{q}^{m} \omega\right)$.

Since $1-\gamma_{k, j} \omega \geqq \max \left\{1-h_{k}^{n} \omega, 1-\hat{h}_{j}^{m} \omega, \omega_{k, j}\right\}$, the above estimate yields

$$
\omega_{k, j} a_{k, j} \leqq \alpha_{k, j}\left(\omega_{k-1, j} a_{k-1, j}+h_{k}^{n} \nu_{k}\right)+\beta_{k, j}\left(\omega_{k, j-1} a_{k, j-1}+\hat{h}_{j}^{m} \hat{\nu}_{j}\right)+\gamma_{k, i} d_{k, j} .
$$

Hence by the induction hypothesis $\omega_{k, j} a_{k, j}$ is majorized by

$$
\begin{aligned}
& \left\|u_{0}^{n}-\tilde{x}\right\|+\left\|\tilde{x}-\hat{u}_{0}^{m}\right\|+\left[\alpha_{k, j} c_{k-1, j}+\beta_{k, j} c_{k, j-1}\right](\|\tilde{y}\|+\rho(T)) \\
+ & \sum_{p=1}^{k} h_{p}^{n} \nu_{p}+\sum_{q=1}^{i} \hat{h}_{q}^{m} \hat{\nu}_{q}+\left(\hat{t}_{j}^{m}-\hat{s}\right) \alpha_{k, j}\left[\kappa^{-1} \rho(T) c_{k-1, j}^{\prime}+\rho(\delta)\right] \\
+ & \left(\hat{t}_{j-1}^{m}-\hat{s}\right) \beta_{k, j}\left[\kappa^{-1} \rho(T) c_{k, j-1}^{\prime}+\rho(\delta)\right]+\gamma_{k, j} d_{k, j},
\end{aligned}
$$

where $c_{k, j} \equiv c_{k, j}(s-\hat{s})$ and $c_{k, j}^{\prime} \equiv c_{k, j}(\eta)$. Therefore, the proof is complete if the sum of the above-mentioned terms is majorized by the right side of (3.9). Applying (3.7) with $\eta=s-\hat{s}$ to the third term, we infer that the third term is not greater than $c_{k, j}(s-\hat{s})[\| \tilde{y}||+\rho(T)]$. Next, set $r=\left|t_{k}^{n}-\hat{t}_{j}^{m}\right|$ and $r^{\prime}=\left|\eta-\hat{h}_{j}^{m}\right|$ in (3.8). Then one has

$$
\gamma_{k, j} d_{k, j} \leqq \beta_{k, j} \hat{h}_{j}^{m} \rho\left(\left|t_{k}^{n--} \hat{t}_{j}^{m}\right|\right) \leqq \beta_{k, j} \hat{h}_{j}^{m}\left[\kappa^{-1} \rho(T) c_{k, j-1}(\eta)+\rho(\delta)\right]
$$

since $r^{\prime} \leqq|\eta|+\left|\hat{\Delta}_{m}\right|<\delta-\kappa$ and $\left|r-r^{\prime}\right| \leqq\left|t_{k}^{n}-\hat{t}_{j-1}^{m}-\eta\right| \leqq c_{k, j-1}(\eta)$. Hence, the 
sum of the sixth through eighth terms is not greater than

$$
\left(\hat{t}_{j}^{m}-\hat{s}\right)\left[\kappa^{-1} \rho(T)\left(\alpha_{k, j} c_{k-1, j}^{\prime}+\beta_{k, j} c_{k, j-1}^{\prime}\right)+\rho(\delta)\right] .
$$

But this is bounded by $\left(\hat{t}_{j}^{m}-\hat{s}\right)\left[\kappa^{-1} \rho(T) c_{k, j}(\eta)+\rho(\delta)\right]$ [by (3.7)]. Thus, it is concluded that (3.9) holds for the pair $(k, j)$.

q.e.d.

We are now in a position to establish the convergence (2.1).

Theorem 3.5. Let $s \in[0, T)$ and $x \in \bar{X}_{\beta, s^{*}}$ Assume that $\left\{A_{t}\right\}$ satisfies condition $\left(H ; X_{o}\right)$, and that a consistent discrete scheme $((D S))_{s}$ for $(D E)_{s}$ exists in $X_{\alpha}$. Then the convergence (2.1) holds in $B([s, T) ; X)$ and the limit function $u$ gives a unique integral solution of $(D E)_{s}$ constrained in $\left\{\bar{X}_{\gamma, t}: s \leqq t \leqq T\right\}$ for some $\gamma \geqq \alpha$.

Proof. Let $s=\hat{s}$ and suppose that $((D S))_{s}$ is the same scheme as $((D S))_{s}$ in Lemma 3.4. Let $\eta=0$ in (3.9). Then, noting that

$$
\exp \left[-\omega\left(t_{k}^{n}-s\right) /\left(1-\omega\left|\Delta_{n}\right|\right)\right] \exp \left[-\omega\left(t_{j}^{m}-s\right) /\left(1-\omega\left|\Delta_{m}\right|\right)\right] \leqq \omega_{k, j}
$$

for $0 \leqq j \leqq N_{m}$ and $0 \leqq k \leqq N_{m}$, (3.9) is written as

$$
\begin{aligned}
& \exp \left[-\dot{\omega}\left(t_{k}^{n}-s\right) /\left(1-\omega\left|\Delta_{n}\right|\right)\right] \exp \left[-\omega\left(t_{j}^{m}-s\right) /\left(1-\omega\left|\Delta_{m}\right|\right)\right]\left\|u^{n}\left(t_{k}^{n}\right)-u^{m}\left(t_{j}^{m}\right)\right\| \\
\leqq & \left\|u_{0}^{n}-\tilde{x}\right\|+\left\|u_{0}^{m}-\tilde{x}\right\|+c_{k, j}(0)[\|\tilde{y}\|+\rho(T)] \\
+ & \sum_{p=1}^{k} h_{p}^{n} \nu_{p}+\sum_{q=1}^{i} h_{q}^{m} \nu_{p}+\left(t_{j}^{m}-s\right)\left[\kappa^{-1} \rho(T) c_{k, j}(0)+\rho(\delta)\right]
\end{aligned}
$$

where $0<\kappa<\delta \leqq T, \max \left\{\left|\Delta_{m}\right|,\left|\Delta_{n}\right|\right\}<\min \{\delta-\kappa, 1 / 2 \omega\}$, and $(\tilde{x}, \tilde{y}) \in A_{s}$. Let $\nu \in(0, T-s)$ and $t \in[s, T-\nu]$. Then there exist $k(n)$ and $j(m)$ such that $t \in\left(t_{k(n)-1}^{n}, t_{k(n)}^{n}\right] \cap\left(t_{j(m)-1}^{m}, t_{j(m)}^{m}\right]$. Note that $\left|t_{k(n)}^{n}-t_{j(m)}^{m}\right| \leqq\left|\Delta_{n}\right|+\left|\Delta_{m}\right|$. Therefore, if $\left\{u^{n}\right\}$ is a sequence of step functions defined as in (2.1), then (3.10) yields

$$
\begin{aligned}
& \exp \left[-\omega\left(t-s+\left|\Delta_{n}\right|\right) /\left(1-\omega\left|\Delta_{n}\right|\right)\right] \exp \left[-\omega\left(t-s+\left|\Delta_{m}\right|\right) /\left(1-\omega\left|\Delta_{m}\right|\right)\right]\left\|u^{n}(t)-u^{m}(t)\right\| \\
\leqq & \left\|u_{0}^{n}-\tilde{x}\right\|+\left\|u_{0}^{m}-\tilde{x}\right\|+(T-s) \rho(\delta)+\sum_{p=1}^{N_{n}} h_{p}^{n}|| f_{p}^{n}\left\|+\sum_{q=1}^{N_{m}} h_{q}^{m}\right\| f_{q}^{m} \| \\
+ & {\left[\kappa^{-1} \rho(T)(T-s)+\|\tilde{y}\|+\rho(T)\right]\left[(T-s+1)\left(\left|\Delta_{n}\right|+\left|\Delta_{m}\right|\right)\right]^{1 / 2} . }
\end{aligned}
$$

Since $\rho(\delta) \downarrow 0$ as $\delta \downarrow 0$ and $\tilde{x}$ can be taken in $D\left(A_{s}\right)$ so that $\|x-\tilde{x}\|$ may be as small as we please, we infer with the aid of the consistency condition $(C)$ that the limit $u(t)=\lim _{n \rightarrow \infty} u^{n}(t)$ exists for $t \in[s, T-\nu]$ and the convergence is uniform on $[s, T-\nu]$ with respect to $t$. Since $\nu$ is arbitrary in $(0, T-s)$, we conclude that the convergence $(2.1)$ holds in $B([s, T) ; X)$ and the proof of the first assertion is complete. The last assertion follows from Theorem 2.4. q.e.d.

A few remarks concerning the above result are in order. 
REMARK 3.1. As mentioned after Definition 2.3 , the family $\left\{\mathcal{A}_{t, \omega}: t \in\right.$ $[0, T]\}$ of extensions of $A_{t}, 0 \leqq t \leqq T$, also satisfies condition $\left(H ; X_{\omega}\right)$ for the same constant $\omega$ as well as the same function $\theta$. So, all of our arguments in this subsection are valid in the second dual $X^{* *}$ even if $A_{t}(t \in[0, T])$ are replaced by the operators $\mathcal{A}_{t, \infty}(t \in[0, T])$. In particular, (3.9) holds for every $(\tilde{x}, \tilde{y}) \in \mathcal{A}_{r, a}$.

REMARK 3.2. As shown in Theorem 2.4 , the limit function $u$ obtained by Theorem 3.5 is extended to a strongly continuous function on $[s, T]$ belonging to the class $\mathcal{I}_{y}[s, T]$. If the initial-value $x$ belongs to $D_{a}\left(\mathcal{A}_{s, \infty}\right)$, then the modulus of continuity for $u$ is determined by the rate of convergence $\rho(r) \downarrow 0$ as $r \downarrow 0$. In fact, let $s=\hat{s}$ and suppose that $((D S))_{\hat{s}}$ is the same scheme as $((D S))_{s}$ in Lemma 3.4. This time, we take $t, t^{\prime} \in[s, T]$ with $\left|t-t^{\prime}\right|<(T-s) / 3$, $\kappa \in(0,(T-s) / 3)$, and $m, n$ with $\max \left\{\left|\Delta_{m}\right|,\left|\Delta_{n}\right|\right\}<(T-s) / 3$, and then set $\eta=t-t^{\prime}$ and $\delta=|\eta|+\kappa+\max \left\{\left|\Delta_{m}\right|,\left|\Delta_{n}\right|\right\}$ in (3.9) with $(\tilde{x}, \tilde{y}) \in \mathcal{A}_{s, a}$. Then we see that $(3.10)$ holds with $c_{k, j}(0)$ in the bracket $[\cdots]$ replaced by $c_{k, j}\left(t-t^{\prime}\right)$. Letting $t_{k}^{n} \rightarrow t^{\prime}$ and $t_{j}^{m} \rightarrow t$ in the estimate (3.10), and then $\kappa \rightarrow 0$ in the resultant inequality, we get

$$
\begin{aligned}
& \exp \left[-\omega\left(t+t^{\prime}-2 s\right)\right]\left\|u\left(t^{\prime}\right)-u(t)\right\| \\
\leqq & 2\|x-\tilde{x}\|+\left|t^{\prime}-t\right|\left[\|\| \mathscr{A}_{s, \infty} x \| \mid+\rho(T)\right]+(t-s) \rho\left(\left|t^{\prime}-t\right|+0\right)
\end{aligned}
$$

for $\tilde{x} \in D\left(\mathscr{A}_{s, \infty}\right)$. Now suppose that $x \in D_{a}\left(\mathscr{A}_{s, a}\right)$. Then a sequence $\left\{\tilde{x}_{l}\right\}$ can be selected in $D\left(\mathcal{A}_{s, \omega}\right)$ such that $\tilde{x}_{l} \rightarrow x$ in $X$ and $\|\left|\mathcal{A}_{s, \infty} \tilde{x}_{l}\right||\leqq| \mathcal{A}_{s, \infty} x \mid+1 / !$ for $l \geqq 1$. Substituting $\tilde{x}_{l}$ into $\tilde{x}$ of (3.11) and letting $l \rightarrow \infty$, we obtain

$$
\left\|u\left(t^{\prime}\right)-u(t)\right\| \leqq \text { const. }\left[\left|t^{\prime}-t\right|+\rho\left(\left|t^{\prime}-t\right|+0\right)\right],
$$

where the constant depends only on $s$ and $x$. (3.12) is also deduced from Proposition 2.5. This type of estimate was first given by Crandall-Pazy [4].

Remark 3.3. If $x \in D_{a}\left(\mathcal{A}_{s, a}\right)$ in Theorem 3.5 , then we have

$$
\|u(t)-x\| \leqq(t-s) \exp [\omega(t-s)]\left[\left|\mathcal{A}_{s, \infty} x\right|+\rho(T)\right] \quad \text { for } t \in[s, T] .
$$

To see this, we first note that Lemma 3.3 is still valid for every $(\tilde{x}, \tilde{y}) \in \mathscr{A}_{\tilde{t}, \infty}$ by the same reason as in Remark 3.1. Let $x \in D_{a}\left(\mathscr{A}_{s, \infty}\right)$ and $\left\{x_{l}\right\}$ a sequence in $D\left(\mathcal{A}_{s, a}\right)$ with the properties as mentioned before (3.12). Then, Lemma 3.3 with $A_{\tilde{t}}$ replaced by $\mathcal{A}_{s, a}$ implies

$$
\begin{aligned}
& \prod_{p=1}^{k}\left(1-h_{p}^{n} \omega\right)\left\|u_{k}^{n}-\tilde{x}_{l}\right\| \\
\leqq & \left\|u_{0}^{n}-\tilde{x}_{l}\right\|+\left(t_{k}^{n}-s\right)\|\| \mathcal{A}_{s, \alpha} \tilde{x}_{l} \|+\sum_{p=1}^{k} h_{p}^{n}\left[\left\|f_{p}^{n}\right\|+\theta\left(t_{p}^{n}, s\right)\right],
\end{aligned}
$$

so that it follows from Theorem 3.5 that 


$$
\left\|u(t)-\tilde{x}_{l}\right\| \leqq e^{\omega(t-s)}\left[\left\|x-\tilde{x}_{l}\right\|+(t-s)\left\|\mid \mathcal{A}_{s, o s} \tilde{x}_{l}\right\|++(t-s) \rho(T)\right] .
$$

Now letting $l \rightarrow \infty$ yields the desired estimate.

REMARK 3.4. To get the convergence (2.1) we approximated the initialvalue $x$ by elements of $D\left(A_{s}\right)$. However (3.9) holds for $r \in[s, T]$ and $(\tilde{x}, \tilde{y}) \in A_{r}$; hence we still have the convergence (2.1) so far as $x$ can be approximated by elements in the union of $D\left(A_{r}\right)(\mathrm{s} \leqq r<T)$. This suggests that the class of "admissible" initial-data can be extended to a larger set than $\bar{X}_{\beta}{ }^{\prime}$. In fact, given an $s \in[0, T)$, let $\boldsymbol{E}\left(A_{s, \beta}\right)$ be the set of all $x \in X_{\beta}$ for which there exist sequences $\left\{r_{l}\right\}$ in $[s, T)$ and $\left\{\tilde{x}_{l}\right\}$ in $X$ such that $r_{l} \downarrow s, \tilde{x}_{l} \in D\left(A_{r_{l}}\right)$ and $\tilde{x}_{l} \rightarrow x$. Then $\boldsymbol{E}\left(A_{s, \beta}\right)$ is closed in $X$ and contains $\bar{X}_{\beta, s^{*}}$. Hence it is sufficient to assume $x \in \boldsymbol{E}\left(A_{s, \beta}\right)$ in order to get the same conclusion as in Theorem 3.5. In other words, the class of admissible initial-data is determined by the family of domains $\left\{D\left(A_{t}\right): t \in[s, T)\right\}$, rather than the single domain $D\left(A_{s}\right)$.

In this connection, it is interesting to note that if $x \in \boldsymbol{E}\left(A_{s, \beta}\right)$ then the first step $u_{1}^{n}$ of the scheme $((D S))_{s}$ converges to $x$. In fact, if $x \in \boldsymbol{E}\left(A_{s, \beta}\right)$ then given an $l \geqq 1$ there exist $r_{l} \in[s, T)$ and $\left(\tilde{x}_{l}, \tilde{y}_{l}\right) \in A_{r_{l}}$ such that $\left|r_{l}-s\right|<1 / l$ and $\left\|\tilde{x}_{l}-x\right\|<1 / l$. So, the application of Lemma 3.2 with $\tilde{t}=r_{l},(\tilde{x}, \tilde{y})=\left(\tilde{x}_{l}, \tilde{y}_{l}\right)$ and $w=0$ yields that $\left(1-h_{1}^{n} \omega\right)\left\|u_{1}^{n}-x\right\| \leqq 2\left\|x-\tilde{x}_{l}\right\|+\left\|u_{0}^{n}-x\right\|+h_{1}^{n}\left[\left\|\tilde{y}_{l}\right\|+\left\|f_{1}^{n}\right\|+\theta\left(t_{1}^{n}, r_{l}\right)\right]$. Therefore $u_{1}^{n} \rightarrow x$ as $n \rightarrow \infty$. In contrast to condition $(T)$ (which asserts that $D\left(A_{t}\right)$ depends upon $t$ in a "forward". sense), this fact means that $x$ is approximated by elements in the union of $D\left(A_{r}\right)(s<r<T)$. In other words, $D\left(A_{t}\right)$ also depends upon $t$ in a "backward" sense under the existence of the scheme $((D S))_{s}$.

REMARK 3.5. As is seen from the argument developed so far, the convergence (2.1) is still valid and the limit is uniquely determined in the class $\mathcal{G}_{y}[s, T]$ even if a Lebesgue null set $N$ is selected and the operator $A_{t}$ is undefined for $t \in N$. In this case $((D S))_{s}$ makes sense only when the sequence of partitions $\left\{\Delta_{n}\right\}$ is chosen so that $\Delta_{n} \subset[s, T]-N$ for $n \geqq 1$. For this type of generalization, see Evans [8].

\section{Construction of evolution operators}

In this section we discuss the construction of evolution operators. We assumed condition $(\boldsymbol{P})$ in Section 1 as one of our basic hypothesis, and condition $(\boldsymbol{P})$ will play an important role in this section.

We recall that $C_{s}(s \in[0, T])$ are the sets defined by (1.7). Our main result is now stated as follows.

Theorem 4.1. Assume that there exists an l.s.c. functional $p: X \rightarrow[0, \infty]$ satisfying $(\boldsymbol{P})$ and $(\boldsymbol{T})$, and that condition $\left(\boldsymbol{H} ; X_{\omega}\right)$ holds for every $\alpha>0$. Suppose 
that given $s \in[0, T), \beta>0$ and $x \in \bar{X}_{\beta, s}$ there exists a discrete scheme $\left(\left\{\Delta_{n}\right\},\left\{\left(u_{k}^{n}\right.\right.\right.$, $\left.\left.\left.v_{k}^{n}\right)\right\},\left\{f_{k}^{n}\right\}\right)$ satisfying conditions (i)-(iii) as mentioned at the beginning of Section 3.1. Then there is an evolution operator $\mathcal{U}$ constrained in $\left\{C_{s}\right\}$ with the following properties:

(a) For $s \in[0, T)$ and $\beta>0$ there exists an $\alpha \geqq \beta$ such that $U(t, s)$ maps $\bar{X}_{\beta, s}$ into $\bar{X}_{a, t}$ for $t \in[s, T]$ and, for each $x \in \bar{X}_{\beta, s}, u(t) \equiv U(t, s) x$ gives a unique integral solution with initial-value $x$ of $(D E)_{s}$ constrained in $\left\{\bar{X}_{a, t}\right\}$.

(b) For $s \in[0, T)$ and $\beta>0$ there exists an $\alpha \geqq \beta$ and the inequality

$$
\|U(t, s) x-U(t, s) \hat{x}\| \leqq \exp \left[\omega_{\omega}(t-s)\right]\|x-\hat{x}\|
$$

holds for $x, \hat{x} \in \bar{X}_{\beta, s}$ and $t \in[s, T]$. Hence $\{U(t, s): s \leqq t \leqq T\}$ is equi-Lipschitz continuous over $\bar{X}_{\beta, s}$.

(c) For $s \in[0, T), \beta>0$, and $x \in D_{a}\left(\mathcal{A}_{s, a}\right) \cap X_{\beta}$, we have

$$
\|U(t, s) x-U(\hat{t}, s) x\| \leqq \text { const. }(s, x)\left[|t-\hat{t}|+\rho_{\alpha}(|t-\hat{t}|+0)\right]
$$

for $s \leqq t, \hat{t} \leqq T$, where $\alpha$ is a number as mentioned in (a), $\theta_{\infty}$ a function in $\mathcal{I}$ provided by condition $\left(H ; X_{\infty}\right)$, and $\rho_{\infty}$ is defined for the $\theta_{\infty}$ by (1.5). If the function $\theta_{\infty}$ is in $\mathcal{I}_{B V}$, then $U(\cdot, s) x$ is Lipschitz continuous over $[s, T]$ with respect to $t$.

Proof. Let $s \in[0, T)$ and $\beta>0$. Given an $x \in \bar{X}_{\beta, s}$, define step functions $u_{n}(\cdot, s ; x):\left[s, t_{N_{n}}\right] \rightarrow X$ by $u_{n}(t ; s, x)=u_{k}^{n}\left(\in X_{\infty}\right)$ for $t \in\left(t_{k-1}^{n}, t_{k}^{n}\right], 1 \leqq k \leqq N_{n}$ and $n \geqq 1$. Then Theorem 3.5 yields the uniform convergence

$$
u(t ; s, x)=\lim _{n \rightarrow \infty} u_{n}(t ; s, x) \quad \text { on } \quad[s, T-\nu]
$$

for every $\nu \in(0, T-s)$, and Theorem 2.4 implies that $u(\bullet ; s, x)$ is a unique integral solution with initial-value $x$ of $(D E)_{s}$ constrained in $\left\{\bar{X}_{\gamma, t}\right\}$ for some $\gamma \geqq \alpha$. More precisely, given $\gamma^{\prime} \geqq \gamma, u(\cdot ; s, x)$ is uniquely determined for the $s$ and $x$ in the class $\mathcal{G}_{\gamma^{\prime}}[s, T]$. Now we set

$$
U(t, s) x=u(t ; s, x) \quad \text { for } \quad t \in[s, T] .
$$

In view of (1.7), $s$ and $x$ are arbitrarily taken in $[0, T]$ and $C_{s}$, respectively. Hence (4.1) provides single-valued operators $U(t, s)(0 \leqq s \leqq t \leqq T)$ such that $U(t, s)$ maps $C_{s}$ into $C_{t}$ and $U(\cdot ; s) x$ is strongly continuous on $[s, T]$ for $s \in[0, T]$ and $x \in C_{s}$. This means that the family $\mathcal{U}=\{U(t, s): 0 \leqq s \leqq t \leqq T\}$ has property (E2) mentioned as in the Introduction. Let $0 \leqq r \leqq s \leqq t \leqq T$ and $x \in C_{r}$. Then we infer from the proof of Theorem 2.4 that $U(\cdot, r) x$ gives an integral solution with initial-value $U(s, r) x$ of $(D E)_{s}$ constrained in $\left\{\bar{X}_{\gamma, t}: s \leqq t \leqq T\right\}$ for some $\gamma$, so that we have $U(t, r) x=U(t, s) U(s, r) x$ by the unicity for weak solutions. This means that $\mathcal{U}$ has property $(E 1)$, and so $\mathcal{U}$ forms an evolution operator constrained in $\left\{C_{s}\right\}$ and has property (a). Property (b) follows from Theorem 3.5 and the estimate (2.7). (The inequality in (b) is also obtained 
directly from the estimate (3.9).) Finally, Assertion (c) is nothing but a combination of restatements of Remarks 3.2, 3.3, Proposition 2.4 and Theorem 2.7.

q.e.d.

Remark 4.1. Suppose in the above theorem that $C_{s}$ is independent of $s$ and $C_{s} \equiv C$. Then $\mathcal{U}$ forms an evolution operator on $C$ with property $(E 2)^{\prime}$. In fact, let $x \in C, 0 \leqq s \leqq t \leqq T$, and $0 \leqq \hat{s} \leqq \hat{t} \leqq T$. Then (1.7) implies that $x \in \bar{X}_{\beta, s} \cap \bar{X}_{\hat{\beta}, \hat{s}}$ for some $\beta>0$ and $\hat{\beta}>0$. Let $\left(\left\{\Delta_{n}\right\},\left\{\left(u_{k}^{n}, v_{k}^{n}\right)\right\},\left\{f_{k}^{n}\right\}\right)$ and $\left(\left\{\hat{\Delta}_{m}\right\},\left\{\left(\hat{u}_{j}^{m}, \hat{v}_{j}^{m}\right)\right\},\left\{\hat{f}_{j}^{m}\right)\{\right.$ be two discrete schemes that are consistent with $(D E)_{s}$ in $X_{\alpha}$ and $(D E)_{\hat{s}}$ in $X_{\hat{\alpha}}$, respectively. Define two sequences of step functions $\left\{u_{n}(\bullet ; s, x)\right\}$ and $\left\{\hat{u}_{m}(\cdot ; \hat{s}, x)\right\}$ in the same way as in the proof of the above theorem. We write $\alpha^{\prime}=\max \{\alpha, \hat{\alpha}\}$ and consider the discrete schemes for the initial-value $x$ in the common set $X_{\alpha^{\prime}}$. Now setting $\alpha=\alpha^{\prime}, \hat{x}=x$, $\eta=t-\hat{t},|t-\hat{t}|<\delta \leqq T$, and $0<\kappa<\delta-|t-\hat{t}|$ in (3.9) and then letting $t_{k}^{n} \rightarrow t$ and $\hat{t}_{j}^{m} \rightarrow \hat{t}$, we infer with the aid of Theorem 3.5 that the inequality

$$
\begin{aligned}
& \exp \left[-\omega_{\omega^{\prime}}(t+\hat{t}-s-\hat{s})\right]\|u(t ; s, x)-u(\hat{t} ; \hat{s}, x)\| \\
& \quad \leqq 2\|x-\tilde{x}\|+(|t-\hat{t}|+|s-\hat{s}|)\left(\|\tilde{y}\|+\rho_{\omega^{\prime}}(T)\right)+(\hat{t}-\hat{s}) \rho_{\omega^{\prime}}(\delta)
\end{aligned}
$$

obtains for $(\tilde{x}, \tilde{y}) \in A_{r, \omega^{\prime}}$, and $r \in[0, T]$. Hence letting $\delta \downarrow|t-\hat{t}|$ yields

$$
\begin{aligned}
& \exp \left[-2 \omega_{\omega^{\prime}} T\right]\|U(t, s) x-U(\hat{t}, \hat{s}) x\| \\
\leqq & 2\|x-\tilde{x}\|+(|t-\hat{t}|+|s-\hat{s}|)\left(\|\tilde{y}\|+\rho_{\omega^{\prime}}(T)\right)+(\hat{t}-\hat{s}) \rho_{a^{\prime}}(|t-\hat{t}|+0) .
\end{aligned}
$$

This means that $U(t, s) x$ is strongly continuous on the rectangular $0 \leqq s \leqq t \leqq T$. If in particular $x \in D_{a}\left(\mathcal{A}_{r, \omega^{\prime}}\right)$ for some $r$, then we see in the same way as in Remark 3.2 that the following inequality holds for $0 \leqq s \leqq t \leqq T$ and $0 \leqq \hat{s} \leqq \hat{t} \leqq T$ :

$$
\|u(t, s) x-U(\hat{t}, \hat{s}) x\| \leqq \text { const. }\left[|t-\hat{t}|+|s-\hat{s}|+\rho_{\omega^{\prime}}(|t-\hat{t}|+0)\right],
$$

where the constant depends only upon $r$ and $x$. Note that $U(t, \cdot) x$ is always Lipschitz continuous over $[0, t]$ with respect to $s$.

\section{References}

[1] Ph. Bénilan: Équations d'évolution dans un espace de Banach quelconque et applications, Thèse Orsay, 1972

[2] V. Barbu: Nonlinear semigroups and differential equations in Banach spaces, Noordhoff Int. Publ., 1976

[3] J. Chambers and S. Oharu: Semigroups of local Lipschitzians in a Banach space, Pacific J. Math. 39 (1971), 89-112.

[4] M. Crandall and A. Pazy: Nonlinear evolution equations in Banach spaces, Israel J. Math. 11 (1972), 57-94.

[5] M. Crandall and L. Evans: On the relations of the operator $\partial / \partial \tau+\partial / \partial$ s to evolution governed by accretive operators, Israel J. Math. 21 (1975), 261-278. 
[6] M. Crandall: A generalized domain for semigroup generators, Proc. Amer. Math. Soc. 37 (1973), 434-440.

[7] M. Crandall and T. Liggett: Generation of semigroups of nonlinear transformations on general Banach spaces, Amer. J. Math. 93 (1971), 265-293.

[8] L. Evans: Nonlinear evolution equations, Israel J. Math. 26 (1977), 1-42.

[9] K. Fukuda, T. Kakitani and S. Oharu: On semigroups and groups of differentiable operators in Banach spaces, to appear.

[10] T. Kato: Nonlinear semigroups and evolution equations, J. Math. Soc. Japan 19 (1967), 508-520.

[11] T. Kato: Accretive operators and nonlinear evolution equations in Banach spaces, Nonlinear Functional Analysis, Proc. Symp. Pure Math., Amer. Math. Soc., 18, Part I (1970), 138-161.

[12] N. Kenmochi and S. Oharu: Difference approximation of nonlinear evolution equations and semigroups of nonlinear operators, Publ. R.I.M.S., Kyoto Univ., 10 (1974), 147-207.

[13] Y. Kobayashi: Difference approximation of Cauchy problems for quasidissipative operators and generation of nonlinear semigroups, J. Math. Soc. Japan 27 (1975), 640-665.

[14] Y. Kobayashi: Nonlinear evolution operators in Banach spaces, Nonlinear Equations in Abstract Spaces (Ed. V. Lakshmikantham), Academic Press, (1978), 113115.

[15] K. Kobayasi and S. Oharu: On nonlinear evolution operators associated with certain nonlinear equations of evolution, Lecture Notes in Appl. Anal., 2, Kinokuniya Book Store Co., Tokyo, (1980), 139-210.

[16] K. Kobayasi and S. Oharu: Nonlinear evolution operators in a Fréchet space, to appear in Japan. J. Math. 10 (1984).

[17] K. Kobayasi, Y. Kobayashi and S. Oharu: Two integral inequalities related to Bénilan's integral solutions of nonlinear evolution equations, Bull. Sci. Engi. Research Labor., Waseda Univ., 93 (1980), 80-87.

[18] K. Kobayasi, Y. Kobayashi and S. Oharu: Nonlinear evolution operators in Banach spaces, II, to appear in Hiroshima Mathematical Journal.

[19] V. Lakshmikantham and S. Leela: Nonlinear differential equations in abstract spaces, Pergamon Press, 1981

[20] R. Martin, Jr.: Nonlinear operators and differential equations in Banach spaces, Wiley-Interscience, 1976

[21] I. Miyadera: Nonlinear semigroups, (Japanese), Kinokuniya Sugaku-Sosho 10, Kinokuniya Book Store Co., Tokyo, 1977.

[22] S. Oharu: On nonlinear evolution operators, (Japanese), Sugaku 30 (1), Iwanami Book Store Co., Tokyo, 55-67.

[23] S. Oharu and T. Takahashi: On nonlinear evolution operators associated with some nonlinear dispersive equations, to appear.

[24] S. Oharu and T. Takahashi: On nonlinear semigroups generated by semilinear operators, to appear.

[25] N. Pavel: Nonlinear evolution equations governed by f-quasi-dissipative operators, Nonlinear Analysis 5 (1981), 449-468.

[26] M. Pierre: Perturbations localment lipschitziennes et continues d'opérateurs maccrétifs, Proc. Amer. Math. Soc. 58 (1976), 124-128. 
[27] M. Pierre: Enveloppe d'un famille de semi-groupes nonlinéaires et équations d' évolution, Seminaire d'Analyse Non-lineaire, Univ. de Besancon, (25) (19761977).

[28] T. Takahashi: Convergence of difference approximation of nonlinear evolution equations and generation of semigroups, J. Math. Soc. Japan 28 (1976), 96-113.

[29] T. Takahashi: Semigroups of nonlinear operators and invariant sets, Hiroshima Math. J. 10 (1980), 55-67.

[30] T. Takahashi: Difference approximation of Cauchy problems for quasidissipative operators and generation of semigroups of nonlinear contractions, Technical Report of National Aerospace Laboratory, TR-419T (1975), 1-15.

Kazuo Kobayasi

Department of Mathematics

Sagami Institute of Technology

Fujisawa, Kanagawa, 251

Yoshikazu Kobayashi

Department of Mathematics

Faculty of Engineering

Niigata University

Niigata 950-21

Shinnosuke Oharu

Department of Mathematics

Faculty of Science

Hiroshima University

Hiroshima 730

Japan 